\title{
ADC Standard IEC 60748-4-3: Precision Measurement of Alternative ENOB Without a Sine Wave
}

\author{
R. Allan Belcher, Senior Member, IEEE
}

\begin{abstract}
A practical analog-to-digital converter (ADC) introduces quantization error in excess of the ideal value and one way of expressing this is by comparing the value of this error with that of an ideal ADC. This comparison is known as the effective number of bits (ENOBs). It is accepted practice to measure ENOB using the signal-to-noise and distortion (SINAD) ratio of a sine-wave input. This paper extends ENOB theory to any arbitrary waveform by including the crest factor of the input signal. It is now possible to apply the ENOB concept to wideband systems. Measuring the SINAD of an arbitrary or multitone waveform with precision normally requires the use of laboratory standard test equipment. However, International Electrotechnical Commission standard 60748-4-3 specifies an alternative method for wideband SINAD measurements that may also be suitable for built-in test. It is essentially a multitone test using two pseudorandom signal sources and is sometimes known as the double comb-filter (DCF) method. This paper demonstrates the requirements for a practical implementation of a DCF-based system for measuring an ENOB of up to 24 bits. It is shown that in a practical application, DCF ENOB and sine-wave ENOB results have similar levels of accuracy, but in the presence of amplitude nonlinearity the differing test signal amplitude weightings cannot fundamentally produce the same ENOB figure. It is shown that DCF ENOB is more representative of communications system performance and therefore extends the use of ENOB to wideband applications.
\end{abstract}

Index Terms-Analog-digital integrated circuits, built-in self-test, International Electrotechnical Commission (IEC) standards, quantization.

\section{INTRODUCTION}

A PURE sine wave or tone is commonly used for testing the linearity and resolution of Analog-to-digital converter (ADC) circuits as it can be made close to ideal using suitable filtering and has the advantage that harmonic measurement equipment is available commercially to check the purity of the source. It also has the advantage that it can be described analytically with ease. As the ADC output signal is a sequence of digital words further analysis can be undertaken using wellknown algorithms such as the fast Fourier transform (FFT). Although the FFT facilitates measurement of sine-wave signalto-noise and distortion (SINAD) ratio, a wider range of tests

Manuscript received August 11, 2014; revised May 14, 2015; accepted June 2, 2015. This work was supported by the Engineering and Physical Science Research Council under Grant EP/D04264X/1. The Associate Editor coordinating the review process was Dr. Niclas Bjorsell.

The author is with the School of Engineering, Cardiff University, Cardiff CF24 3AA, U.K. (e-mail: belchera@cardiff.ac.uk)

Color versions of one or more of the figures in this paper are available online at http://ieeexplore.ieee.org.

Digital Object Identifier 10.1109/TIM.2015.2450296 are required to ensure that an $\mathrm{ADC}$ will be suitable for particular applications. Unfortunately, variations in the method used to measure SINAD can change the result so it was not easy to compare the performance of one manufacturer's ADC with that from a different manufacturer. This obviously can cause a problem for the system designer. For this reason, it became necessary to define and standardize internationally a range of ADC test methods. This paper is concerned with wideband effective number of bits (ENOBs).

In essence, ENOB compares the performance of a practical $\mathrm{ADC}$ with that of perfect ADC of the same resolution in bits. ENOB is a measure of the rms error between the input-output transfer characteristics of an ideal ADC and a practical ADC of the same resolution. This comparison could, in principle, be measured simply using a test signal with a uniform amplitude probability distribution function (APDF) such as a ramp signal. However, the linearity of a ramp waveform is limited by the linearity of analogue circuits and unlike a sine wave its APDF cannot be improved by filtering. In practice, ENOB is measured with a sine wave as its APDF is known and reproducible, but it will not necessarily produce the same rms error and ENOB as a ramp waveform. As the APDFs are different, amplitude nonlinearity can result in a sine-wavebased ENOB being worse than a ramp-based ENOB when the signal peaks are distorted. It is therefore important to take this APDF dependency of ENOB into account when comparing a sine-wave-based ENOB with one measured using an alternative test signal.

In 2006, the publication of the International Electrotechnical Commission (IEC) standard IEC 60748-4-3 [1] provided for wideband measurement of ENOB with an alternative test signal. It includes input from IEEE [2] and the national standards bodies of IEC member countries worldwide. It is important to note that IEC standards are industry led. As IEC standards are world standards they may be adopted in legislation, enabling potential barriers to trade to be overcome. In contrast, other standards, though in use worldwide may be voluntary. It may be useful here to compare how IEEE and IEC standards are written. IEC standards conform to ISO/IEC Directives governing the drafting and structure of international standards [3], [4] and IEEE standards are written to conform to the IEEE standards organization style manual. Both IEEE and IEC standards contain normative elements which define what must be done to comply with regulatory requirements. Guidance as to how to understand the concepts presented is provided by informative elements. IEC provides guidance or 
informative detail through its technical report route which requires only the approval of an IEC technical committee. An IEEE standards committee may choose to write a standard that contains a significant amount of detail of an informative nature. In contrast, an IEC standards committee might instead decide to publish detailed informative text as an IEC technical report. For anyone who is not aware of this difference in policy, an IEEE standard may often have more informative text and therefore appear to be more detailed than an IEC standard.

IEEE has been represented at IEC through IEEE members being nominated by their national standards organization; this is the process for new IEC standards work to be started. Organizations such as IEEE can apply to IEC for liaison status. This enables a member of an IEEE standards committee to be an observer at an IEC meeting. As in IEEE 1241, IEC 60748-4-3 defines the dynamic performance of an ADC by evaluating its performance with reference waveforms such as linear ramp, square, and one or more sine waves. However, the IEC working group also considered documents from other IEC countries and arrived at a consensus.

For wideband measurements it specifies a test signal of two pseudorandom signal sources which in effect is a multitone test signal. This is sometimes known as the double combfilter (DCF) method. As the method can be implemented using a small number of logic elements and does not require FFT analysis, it is particularly suitable for built-in-test applications. This section of the standard specifies results that relate only to the wideband noise-like test signal. At the time this standard was written, there was no evidence to indicate that measurements made with this DCF test system could be directly related to the ENOBs of a sine wave, and for that reason the test was intended for routine rather than type-approval use.

\section{A. Type-Approval Testing}

When the performance of an ADC is determined using commercial test equipment that meets the requirements of a standards laboratory the cost, accuracy, and repeatability required makes this a time consuming and expensive process. Measurements such as these are usually only performed to ensure that the type of ADC, when performing correctly, is able to meet stringent performance requirements of the manufacturer or customer.

\section{B. Routine Testing}

Manufacturing faults can occur that result in the performance of the ADC to be grossly in error and it is this type of low cost measurement that may be made routinely in a production test environment or for health monitoring of a system. A routine test method has significantly shorter test duration and lower precision than a type approval test as it need only be sufficient to detect gross faults.

What is required is a test of short duration that does not require expensive components but has the potential to be used for type-approval testing. This is the application area of the DCF test method. It has long been understood that nonlinearity measured with a sine wave may not be a true indication of the performance with real signals as these have a different APDF and is closer to Gaussian noise. It is therefore unlikely that they will both generate the same rms nonlinearity error. There is a need to establish situations where sine and DCF ENOB may be expected to be in close agreement and when this is not likely. This will assist in deciding which ENOB is a better indicator of system performance. This standard [1] does not make a specific recommendation for the most appropriate configuration of pseudorandom signal generator or the required digital filters. The theoretical analysis and practical design choices for a DCF test system presented in this paper will show how this DCF method can be implemented in practice with the key factors that limit measurement precision established. This will enable the DCF method to be used for both routine and type-approval purposes.

A demonstration of this DCF method was provided for the IEC working group in the form of .wav format stereo audio files. These audio files were employed in the test and simulation results described in this paper and are available on request. The following sections in this paper present the case for using the DCF method in applications where a precision measurement of ENOB is required but where it is impractical to use a sine-wave-based method or where a sine wave is not a good representation of performance with real wideband signals. The case is presented progressively in the following sections.

Section II uses the test waveform independent definition [2] of ENOB to compare sine-wave ENOB accuracy with ENOB from other test waveforms. The noise and distortion (NAD) concept in the ENOB definition is refined to cover parameters in the measurement of ENOB that limit precision or add uncertainly in measurement. NAD is shown to include ideal quantizing noise power, additional error power due to imperfect quantization, and power added by random noise sources and sources of measurement uncertainty. Insufficient code occurrence density can produce uncertainty in the measurement of ideal quantizing noise. The concept of this being represented by ENOB is introduced. A general equation for ENOB is derived based on the SINAD and crest factor or peak-to-rms ratio of an arbitrary test waveform. The importance and difficulties in generating and using a test signal representative of a real communications signal is discussed.

Section III describes the DCF method and presents two practical implementations of the test signal generator. The key practical limitations and advantages of these generators are described. Central to the DCF method is the concept that, with appropriate choices of DAC sampling frequency and comb base frequency, all intermodulation and harmonic products of a nonlinear DAC can be aliased back on to the comb spectrum so are hidden. Nonlinearity in any following amplifier will generate products that simply fall onto the test signal frequency locations so are hidden or are outside the test bandwidth. These can be removed by suitable low-pass filtering. Sensitivity to amplitude nonlinearity occurs only after the two interleaved comb spectra are summed.

As the power loss through a comb filter varies with the power spectral density of the input signal, a precision measurement of ideal quantization noise is required. Gain correction factors were calculated for each filter, based on the assumption 
that the power loss experienced by a white noise input signal would be a good approximation. The following questions are also addressed concerning the rms ideal quantizing noise $Q$. Is the crest factor of aliased quantizing noise $4.77 \mathrm{~dB}$ and what crest factor should be expected for $Q$ at the output of the DCF measurement filter chain? In the following text, this chain will simply be referred to as a measurement filter and is comprised of comb and low-pass filters (LPFs) and is described in Section III-A2. It was possible that insufficient code density in the test signal could alter the crest factor of $Q$ and it could also be changed by the measurement filter. This problem was investigated by simulation and generation of the true ideal quantizing error waveform by an approach similar to curve fitting. This gave the ideal quantizing noise waveform present at the input to the comb filters and its measured crest factor was $4.77 \mathrm{~dB}$. When this $Q$ waveform was passed through the DCF measurement filter its crest factor was increased to the same value as in a DCF measurement of $Q$, confirming that the measurement filter alone caused the increase in crest factor. As this waveform may not be true noise (i.e., random) or white (i.e., uniform power spectral density), the calculated white noise loss (see Section III-A2) of each filter element may not be accurate. Power loss of the quantizing error through the comb and LPFs was instead measured directly with the real quantizing error signal and a gain correction value determined.

Section IV presents a simulation of potential sources of ENOB measurement error with ideal and practical ADCs. It includes ideal ADCs of 24-8 bits, two practical ADC characteristics of 8 bits with segment boundary transition errors and 10 bits with a low-order polynomial error, both with added noise. Routine tests are usually of short duration. Some insight into the comparative sensitivity of the accuracy of DCF and sine results to test duration was obtained using no more than $2 \mathrm{~s}$ of test signal. Results indicate that the DCF ENOB is more sensitive to segment boundary transition errors than a sine wave, but is less sensitive to low-order polynomial error than a sine wave. This supports the view that a DCF measurement of ENOB is a better representation of ADC performance in a practical application.

Section $\mathrm{V}$ presents the results of applying sine and DCF test methods to a professional quality 24-bit ADC-DAC audio interface. Results demonstrate that in a practical 24-bit system where no external filtering is used between audio outputs and audio inputs, random noise is the dominant source of error; sine and DCF methods then measure SNR with equal precision but the sine-based ENOB is less than the DCF-based ENOB.

\section{Measuring EfFective Number of Bits}

The precision or degree of accuracy of a measurement of ENOB for an ADC is ultimately limited by imperfections in the test signal source and inaccuracy or uncertainty in the method of measurement. A sine wave is the test signal of choice for type-approval testing. Although most audio signal sources provide total harmonic distortion (THD) in the range -100 to $-120 \mathrm{~dB}$, the need for increased performance has driven commercial innovation and an increase to better than
$-140 \mathrm{~dB}$ is now available through a harmonic cancellation method [5]. European Metrology Institutes have approached this problem by employing superconducting techniques to produce a Josephson Junction based DAC through the Q-wave project [6]. Publically available results so far indicate that the theoretical limit of an ideal low-order delta sigma DAC may have been achieved [7], [8]. Superconducting bandpass filters are used in mobile communications base stations and offer a further way to reduce THD [9]. With all this recent activity in parallel areas of research and development one might wonder where the true limit of THD could be 10 years from now.

However, it has long been recognized that a sine wave is not representative of real wideband signals present in communication systems [10]. Modern communication systems have real signals with well-defined peak-to-rms power ratios or crest factors. Typically, these crest factors are at least $9 \mathrm{~dB}$ so the $3 \mathrm{~dB}$ of a sine wave is not a good match and may over value the importance of nonlinearity at signal peaks and undervalue it elsewhere. It is important to note that $\mathrm{dB}$ crest factor is numerically the same as decibel peak-toaverage power ratio (PAPR). PAPR is a key parameter for wideband digital communication systems [11]. The ENOB figure is influenced by the APDF of the particular test signal but signals with similar crest factors do not necessary have the same APDF. It is possible, when amplitude nonlinearity is present, that two signals with the same PAPR but differing APDFs could give different ENOB figures. It is therefore important to be able to describe the APDF of the ENOB test signal with sufficient accuracy to ensure repeatability. The dual pseudorandom binary sequence (PRBS) waveform reported here addresses this issue as it has a reproducible APDF [12] which, although close to a truncated Gaussian distribution, can be modeled by a five harmonic Fourier series. With 95000 samples and 16-bit quantization the residual rms fitting error was equivalent to 13 bits. This wideband ADC nonlinearity testing problem has also been addressed by others using statistical and curve fitting methods to both random and standard pseudorandom signals with limited success [13], [14].

For routine testing, the noise power ratio (NPR) test method has long been specified for communications systems [15], [16]. It uses band limited random noise with measurements made using a spectral notch in the test signal [17]. The fundamental assumption made with this NPR test is that all the noise and nonlinearity products are spread uniformly across the bandwidth of the test signal so that the total error power can be estimated from the ratio of measurement bandwidth to total bandwidth. In practice, the difficulty in generating a deep enough spectral notch in the random noise test signal has restricted it to routine tests of low-resolution ADCs. NPR is useful from the point of view of it being a wideband test with an APDF closer to that of real communication signals. Pseudorandom noise is periodic but if the sequence length is sufficiently long, it will approach a truncated Gaussian APDF as the number of samples is increased but there is then a tradeoff between test time and accuracy of the APDF. As the distribution is truncated it will have a well-defined peak value so the risk of unexpected overload can be minimized. The availability of low-cost programmable arbitrary waveform 
generators offers an alternative way of generating noise like test signals. Digital signal processing algorithms can find a solution where a relatively small number of harmonically sine waves can, through changing the phase relationship of each sine wave, generate a waveform that has APDFs that approximate to either truncated normal or uniform characteristics [18]. This technique may be particularly useful when it is necessary to minimize the crest factor of a multitone test signal [19].

A bandpass version of a multisine test signal is a preferred test signal for nonlinear model testing of microwave amplifiers as its APDF can be adjusted to be a good representation of the near Gaussian APDF of real signals in microwave communications systems [10]. The generation process is similar to that described in [18] where a comb of equally spaced frequencies is generated but [10] has a precise mathematical analysis of the APDF and a prediction of the power spectral density of out of band products generated by amplitude nonlinearity. Often the out of band products may be removed by channel filters so are not available. In that case an alternative multitone approach is employed for in-band measurements and is known as the multitone power ratio (MTPR) method [2]. Typically, this is used to test bandpass signal transmission modems that have a real signal made up of multitones. The test signal may have up to 256 equally spaced tones chosen to model the real signal. Some of the tones will be eliminated to give an opportunity for in-band intermodulation products to appear at those positions. Unfortunately, the sensitivity of the measurement is limited by the amount of distortion power that can be generated in the empty positions so measurements require DFT-based noised measurements rather than notch filters. The dynamic range is limited by the linearity and resolution of the AWG as the missing tones required in the harmonic sequence can be added by the AWG limiting ADC measurement dynamic range [20]. This limitation has restricted this method to routine testing and it provides a figure of merit rather than a SINAD or ENOB. The use of equally spaced multitones for NPR measurement has been proposed for the measurement of in-band nonlinearity in RF circuits [21]. This is essentially the same as multiple measurements of MTPR: a sequence of measurements is made with the position of the missing tone changed. A sequence of DFT measurements calculates the power in each slot. However, the practical results presented indicate that this is not a high-resolution method and is intended for RF systems where third-order nonlinearity is dominant. The main differences between multitone NPR (as in [21]) and MTPR are: in [21], a smaller number of tones are used, the preferred APDF is closer to Gaussian, and the position of the missing tone is moved across the passband with NPR measurements made at each new position.

\section{A. Error Sources in the Measurement of ENOB With an Arbitrary Waveform}

Routine tests simply indicate the presence of error power due nonlinearity and noise and are useful in indicating the onset of a fault. However, each method of measurement has practical limitations. The following puts forward a relationship that will enable the significance of potential error sources to be evaluated in measuring ENOB with any arbitrary waveform. The basic definition of ENOB [2] is reproduced in

$$
\mathrm{ENOB}=N-\log _{2}\left\{P_{\mathrm{ENOB}} / Q\right\}
$$

where

$N$ specified number of bits in the ADC;

$P_{\text {ENOB }}$ total rms error;

$Q \quad$ rms ideal quantization error.

Practical measurements of ENOB require measurements of amplitude nonlinearity represented by integral nonlinearity error, noise due to differential nonlinearity and quantizing error, and random or thermal noise due to clock jitter or active components. We will define amplitude nonlinearity by first fitting a polynomial to the ADC input-output characteristic. The rms error between this polynomial and a best fit straight line we will define as $P_{\mathrm{INL}}$. This error will be an indication of the component of integral nonlinearity that generates products that are highly correlated with the test signal. $P_{\mathrm{INL}}$ will therefore be related to harmonic and intermodulation products. We will define $P_{\mathrm{DNL}}$ as the rms error left after subtracting the best fit polynomial from the ADC transfer characteristic as this will represent products that are not highly correlated with the test signal. For the purpose of illustrating limits to ENOB test methods, we will assume the various error sources are independent and define $P_{\mathrm{ENOB}}$ as

$$
P_{\mathrm{ENOB}}=P_{\mathrm{INL}}+P_{\mathrm{DNL}}+P_{r}+P_{m}+Q
$$

where $P_{r}$ is the added noise due to random sources such as amplifiers and jitter. $P_{m}$ is the rms error inherent in the method of measuring the other parameter values, e.g., due to insufficient number of samples causing missing codes or the test signal amplitude not exercising the full-coding range of the ADC.

In a simulation of a practical ADC with unwanted noise and quantization errors, the total rms error can be determined simply by subtracting an ideal floating point version of the arbitrary waveform from the output of the practical ADC. Care must be taken to ensure that gain and offset of this ideal test waveform is adjusted so that only the true error $P_{\text {ENOB }}$ remains. Error measurements based on the need to precisely subtract a reference waveform from a quantized waveform can present difficulties as illustrated by the number of solutions published to overcome problems with sine-wave curve fitting [22]. Imperfect sine-wave fitting increases the rms residual error and therefore provides an incorrect ENOB. If an ideal $\mathrm{ADC}$ is simulated with quantizing interval $\delta$ then the subtraction process will result in the actual quantizing error waveform being produced; its mean square value should be $\delta^{2} / 12$ if the approximation [23] that the quantizing noise waveform can be represented by a variable slope sawtooth holds. As the peak amplitude of this ideal quantizing error waveform is $\delta / 2$ and its rms amplitude is $\delta / \sqrt{ } 12$ it follows that its crest factor is $\sqrt{ } 12 / 2$ or as a decibel ratio, $20 \log _{10}(\sqrt{ } 12 / 2)=4.77 \mathrm{~dB}$. This result will be referred to later in deriving a relationship for the ENOB of an arbitrary waveform. 
All these sources of error are present in a sine-wave-based ENOB as well as an ENOB based on an arbitrary waveform. The error $P_{m}$ may be more significant for ENOB based on coherent measurements. For a given number of samples and ADC bits, it is well known that the number of missing codes can be minimized by choosing a relatively prime ratio of test signal frequency to the number of samples [24]. A correct choice will result in the code occurrence histogram having a distribution similar to that of an ideal waveform, but will have missing codes if the number of samples is not sufficient to hit each code at least once. This effect can be seen with any arbitrary waveform by generating sets of code occurrence histograms where the number of ADC bits is varied.

In principle, any arbitrary periodic waveform could be used to measure wideband ENOB, provided the method of measurement is able to measure all the individual components of $P_{\mathrm{ENOB}}$. In the limit, the method must be able to measure the quantizing error of an ideal ADC with the same precision as a sine-wave curve fit. For any arbitrary multitone waveform, this can be achieved in a simulation by adjusting gain and offset to obtain a residual error waveform. However, in a practical wideband test situation frequency dependent gain, offset, and phase errors make this subtraction process inaccurate. Frequency-domain analysis can to some extent overcome this limit using a DFT-based SINAD measurement but unless the multifrequency test signal captured has complete cycles of each frequency component, leakage will occur. This can be compensated for by windowing but this also introduces inaccuracy in the measurement of both signal frequencies and power. If the test signal generation method suffers from these sources of error then they must be low enough not to affect the measurement of ADC ENOB.

For a sine wave, leakage can be avoided by using a test frequency that is synchronous with the sampling frequency and passive bandpass filtering at the input to the ADC can minimize the amplitude of harmonics and noise. If the sine wave is digitally synthesized then noise due to jitter in the DAC clock can be minimized by locking the ADC and DAC clocks to a low noise common clock. Noise within the passband of the filter must be minimized by ensuring that the noise due to differential nonlinearity and quantizing is sufficiently low. Having addressed the potential sources of error in a measurement of ENOB the next step is to derive a simple relationship for the ideal signal to quantizing noise ratio (SQNR) that applies to any arbitrary waveform and from this define the ENOB of any waveform in terms of SINAD and crest factor. The crest factor or PAPR of a waveform is a key parameter in a communications system [19] as it determines the maximum system signal to noise ratio and therefore the bit error rate and service area. The equation derived next widens the scope of ENOB by making it easier to apply to wideband communication systems.

\section{B. Calculation of SQNR of Any Arbitrary Waveform}

The aim of the next part of this analysis is to derive an equation for quantizing error in a linear $n$-bit ADC based on the crest factor of the test signal. Amplitude and power are related by the crest factor $K$ or peak-to-rms amplitude ratio. For an $n$-bit $\mathrm{ADC}$ with linear quantization and quantizing interval $\delta$, the maximum peak signal $A$ is

$$
A=\delta \cdot 2^{n-1} .
$$

The mean square value $P$ of an input signal of peak amplitude $A$ with crest factor $K$ is

$$
P=(A / K)^{2} \text {. }
$$

If $q$ is the mean square amplitude of the quantizing error then

$$
q=\left[\delta^{2} / 12\right] .
$$

Equation 5 is an approximation based on the assumption that the quantizing error can be represented by a variable slope sawtooth [23]. It also requires that the signal amplitude is much larger than $\delta$. As the quantizing error is the only source of NAD in an ideal linear ADC this allows us to produce an equation for SQNR in terms of $n$

$$
\mathrm{SQNR}=P / q=\left(\left(\delta \cdot 2^{n-1}\right) / K\right)^{2} /\left(\delta^{2} / 12\right) .
$$

Next, $\delta$ cancels out so

$$
=\left(2^{2 n-2}+K^{-2}\right) \cdot 12 .
$$

In decibels

$$
\begin{aligned}
\operatorname{SQNR}(\mathrm{dB})= & 10 \log _{10}\left(\left(2^{2 n-2}+K^{-2}\right) \cdot 12\right) \\
= & n \cdot 20 \log _{10} 2-20 \log _{10} 2 \\
& -20 \log _{10} K+10 \log _{10} 12 .
\end{aligned}
$$

Let

$$
\begin{aligned}
C & =20 \log _{10} K \\
& =6.02 n-6.02-C+10.79 .
\end{aligned}
$$

Hence, for an arbitrary waveform of crest factor $C$ in decibels the SQNR in decibels is given by

$$
\mathrm{SQNR}=6.02 n+4.77-C .
$$

It is interesting to note that $4.77 \mathrm{~dB}$ was shown earlier to be the crest factor of quantizing error.

As $C=3.01 \mathrm{~dB}$ for a sine wave, substituting in (7) we have the well-known result

$$
\mathrm{SQNR}=6.02 n+1.76 \mathrm{~dB} .
$$

When imperfections in the ADC are included SQNR is replaced by SINAD then ENOB $n_{e}$ is related to SINAD by

$\operatorname{SINAD}=6.02 n_{e}+1.76 \mathrm{~dB}$. Or $n_{e}=(\operatorname{SINAD}-1.76) / 6.02$.

Hence, for any arbitrary waveform

$$
n_{e}=(\operatorname{SINAD}+C-4.77) / 6.02 .
$$

However, to apply this equation it is first necessary to devise a measurement method that can, as a minimum, measure with accuracy the value of the ideal SQNR of an arbitrary waveform. This aim has so far proved impossible to achieve (with well-known test signals) with the same precision as a sine wave. With a sine wave, the total harmonic power indicates 
the value of $P_{\mathrm{INL}}$ and therefore harmonic distortion in the test signal source limits the threshold of this measurement and contributes to $P_{m}$. Nonlinearity and quantizing error in the DAC therefore limits $P_{m}$ for sine-wave testing but can be determined by spectral analysis. Random or spurious noise sources will also increase $P_{m}$. On the measurement side sinewave curve fitting algorithms and DFT power measurement algorithms are potential sources of $P_{m}$.

\section{Potential Sources of Measurement Error in Making ENOB Measurements With an Arbitrary Waveform}

Practical implementations of the DCF method are described in Section III but the potential sources of $P_{m}$ are as follows.

In the test signal generator they include the following.

1) Noise in the test signal source: this can be minimized using low noise amplifiers and low jitter clocks.

2) Aliasing of out of band harmonics, intermodulation products, and quantizing error of the test signal into the spectral gaps between the teeth of the test signal.

The analog dual PRBS generation method described in Section III avoids this problem completely as the harmonic relationship between 1-bit DAC sampling frequency and PRBS base frequency ensures that all products of DAC nonlinearity are aliased back on to the test signal comb of frequencies and not to values that fall between the teeth of the test signal. Nonlinearity in circuits that follow the DAC will produce in band products that are hidden by the test signal and out of band products that can be removed by low-pass filtering. The mathematical proof for this is given in Section III. Sensitivity to amplitude nonlinearity starts only when the two PRBS waveforms are summed. A dual multibit DAC solution has the advantage that a digital LPF eases the requirements of the analog filter design. It may be impractical to operate a multibit DAC at the PRBS effective sampling rate so subsampling is required to lower the sampling rate of each DAC. However, unless the clock rate of each DAC is a harmonic of its associated PRBS base frequency, insufficient stop band attenuation of the digital filter, and DAC nonlinearity will cause aliased products of the test signal to appear between the teeth of the test signal. When the same sampling rate is used for each DAC it may not be easy to arrange for both PRBS base frequencies to be subharmonics of the sampling rate. However, the effect of DAC and amplifier nonlinearity can be determined by comparing measurements with both test signals. This will then indicate if it is necessary to hide these aliased products by using different subsampling frequencies for each DAC. In some configurations, such as the audio one described in Section V, it is not possible to clock each DAC at nonstandard rates so the linearity performance of each DAC will contribute to $P_{m}$ and its significance must be determined. However, if it is less than the noise level it may not be a significant contribution to a SINAD result. The first step is to set the PRBS base frequency to a subharmonic of $48 \mathrm{kHz}$. This will cause all products of DAC nonlinearity to appear on the test signal teeth and so be hidden. A comparison of the power between the teeth of the test signal with the two PRBS base frequencies will reveal the contribution of DAC nonlinearity, noise and quantizing error to $P_{m}$.

3) Linearity of the network that sums the PRBS signals is an ultimate limit to the performance. This can be achieved using a passive network with sufficient isolation between each path to reduce nonlinear cross talk (caused if the output impedance of an amplifier is changed when the output is driven from another source) to an acceptable level. If the ADC under test has a balanced input then this may be used instead to combine two unbalanced PRBS test signals.

In the test signal analyzer the main sources of $P_{m}$ are the following.

4) The assumption that intermodulation products, quantizing error, and random noise have equal net power loss through the measurement filter.

5) Aliasing of error signals above the stop band of the $20 \mathrm{kHz}$ digital LPF.

DCF waveform measurements of power and crest factor require an accurate baseband waveform. Aliased signals above the digital filter cutoff frequency can appear in the baseband. The out of band attenuation of this filter ultimately limits measurement precision and for 24-bit precision it has been determined that this should be at least $160 \mathrm{~dB}$ : although this figure may seem high this performance was easily achieved using a standard digital filter design program. Section III describes practical implementations of the DCF method. It will be shown that by careful selection of the base frequencies and number of PRBS signals it is possible to estimate $P_{m}$ and measure $\left(Q+P_{\mathrm{DNL}}+P_{r}\right)$, and $P_{r}$ with accuracy comparable with that of a sine wave. The $P_{\text {INL }}$ rms value for a sine-wave $P_{\mathrm{ENOB}}$ is influenced by highest code density at the end points of the ADC characteristic. In contrast the $P_{\mathrm{INL}}$ rms value of DCF ENOB is influenced by highest code density at the center of the ADC characteristic. It is therefore most unlikely that these $P_{\mathrm{INL}}$ rms values will agree and is a fundament reason why practical measurements of ENOB with the DCF method may not give the same ENOB as a sine wave.

\section{Possible Alternative Arrangements for Arbitrary Waveform Generation}

The investigations described in the following sections of this paper are restricted to a particular set of PRBS test signals. However, other choices of base frequency and sequence lengths may also be considered. PRBS base frequencies must be chosen to maximize the power recovered by the comb filters and to minimize the number of spectral lines common to the set of PRBS test signals. A single and a dual PRBS test signal has been chosen as this arrangement can be implemented in a dual DAC system or in a dual channel arbitrary waveform generator or simply as a shift register arrangement. The PRBS test signal upper bandwidth is limited mainly by the maximum clock rate of a shift register so is easily more than $10 \mathrm{GHz}$ with discrete components and an integrated circuit $80 \mathrm{GHz}$ PRBS has been reported [25]. Although in principle, more than two PRBS signals could be used, multichannel DACs and arbitrary waveform generators are not routinely available. 


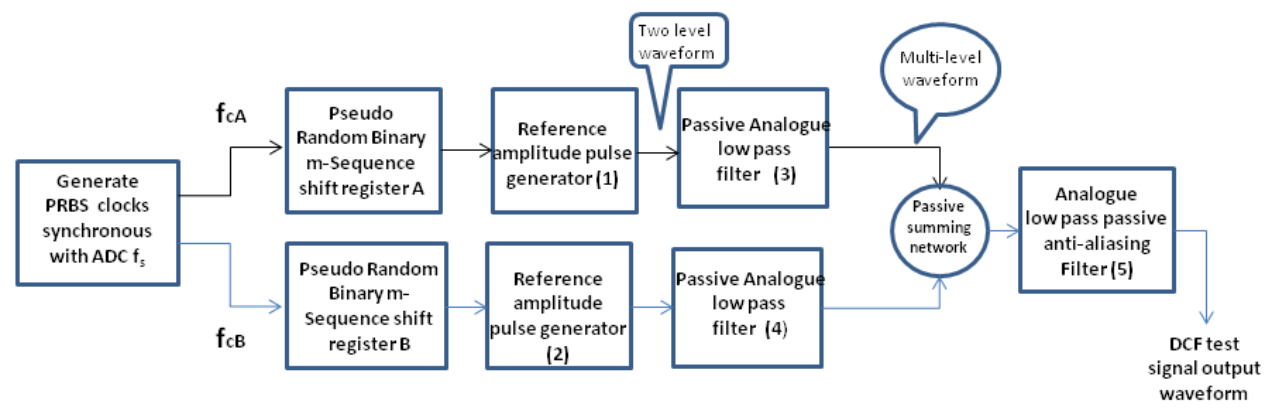

Fig. 1. Analog synchronous DCF test signal generator. Notes: block numbers (1) and (2) amplify the analog two-level waveform from each shift register and sets the peak-to-peak value of each, (3) and (4) are LPFs with bandwidths of approximately $0.1 f_{\mathrm{cA}}$ and $0.1 f_{\mathrm{cB}}$, and (5) is an LPF with a bandwidth of half the ADC Nyquist sampling frequency $f_{s}$.

Increasing the number of PRBS generators may offer advantages such as enabling the frequency of the common clock to be reduced. Other issues to be investigated in the future could include modifying the comb response so that it has a more uniform passband. White noise loss in the measurement filter is approximately $3 \mathrm{~dB}$ for each comb filter so increasing the number of PRBS signals will increase spectral shaping of the quantization and nonlinearity error, change the crest factor of the test signal and perhaps result in a loss of sensitivity to some type of nonlinearity. The main problem to consider is the need to minimize the number of harmonics of base frequencies that coincide as this can create peaks in the spectrum. This could increase the crest factor to a point where the test signal duration would have to be increased to maintain a minimum code density at the peaks.

\section{DCF METHOD}

The DCF method was devised in 1977 at the Engineering Research Department of the British Broadcasting Corporation (BBC) [26]. Its aim was to solve a long standing problem in broadcasting: how to make a measurement of audio circuit nonlinearity that could predict with accuracy the audible impairment of sound program signals. This DCF test system achieved estimates of audible impairment much superior to those of sine-wave-based measurements. However, it used digital circuitry which at the time was much more expensive than analogue circuitry so was essentially a research tool. It was also used to measure quantizing distortion and quantizing error [27] in the evolving BBC digital audio systems. This application is described in more detail elsewhere [28]. Both the audio and ADC applications can be now implemented using just the hardware of a personal computer and application software. However, this paper is concerned only with the measurement of ENOB through precision measurement of the independent error signals that makeup this figure. If an ideal $\mathrm{ADC}$ is considered then the error is referred to as quantizing noise [23].

There are several design problems to address when implementing a practical DCF test system. These have been outlined in Section II and this section will describe two practical methods for implementing the test and give a set of parameter values suitable for testing the highest resolution ADCs available at present, i.e., 24 bits.

\section{A. Practical Implementation of DCF Test System}

1) Test Signal Generation: A simplified block diagram for a digital implementation of the DCF test signal is given in Fig. 1. In Fig. 1 (and later in Fig. 2), more than one arrangement is possible for the block generate PRBS clocks synchronous with ADC $f_{s}$. The details depend on whether the ADC and test signal generator are synchronized by means of a common clock or by phase locking independent clocks. These details are omitted for clarity. PRBS clock frequencies $f_{\mathrm{cA}}$ and $f_{\mathrm{cB}}$ must be synchronous with the ADC sampling frequency $f_{s}$.

Each PRBS requires a shift register and exclusive-OR gate to produce a maximum length or $m$-sequence. The exclusiveOR gate is connected to specific taps on the linear shift register and these positions uniquely define the phase, frequency, and relative amplitude of each spectral line. A generator polynomial [29] defines the length of the linear shift register and positions of the exclusive-OR taps required to produce the sequence. The generator polynomial is sufficient to enable the PRBS signal to be reproduced in hardware or firmware. An $m$-sequence, with an appropriate generator polynomial, can produce a comb spectrum with harmonics all of equal amplitude and multiples of a common base frequency [29]. The spacing $f_{m}$ of the comb of frequencies generated by an $m$ bit shift register with clock frequency $f_{c}$, is

$$
f_{m}=f_{c} /\left(2^{m}-1\right) \text {. }
$$

When a PRBS is applied to an LPF with a bandwidth of less than $0.1 f_{c}$ it reconstructs a multilevel waveform that is noiselike and the number of frequency components is set by the ratio of PRBS clock frequency to LPF bandwidth. The crest factor of an arbitrary waveform is set by the number of frequency components and relative phase and amplitude of each. Relative phase and amplitudes of the spectral components of a PRBS waveform are set by the generator polynomial described in Section III-A1a. The crest factor of a PRBS waveform is therefore predictable and repeatable.

For coherent testing, PRBS generators $A$ and $B$ have a common clock that is an integer multiple of the ADC sampling rate. To illustrate the practical issues involved an ADC sampling rate of $48 \mathrm{kHz}$ will be assumed. As the base frequencies of $A$ and $B$ are each subharmonics of the common clock any nonlinearity in the amplification of each PRBS will simply generate new harmonics or intermodulation products 


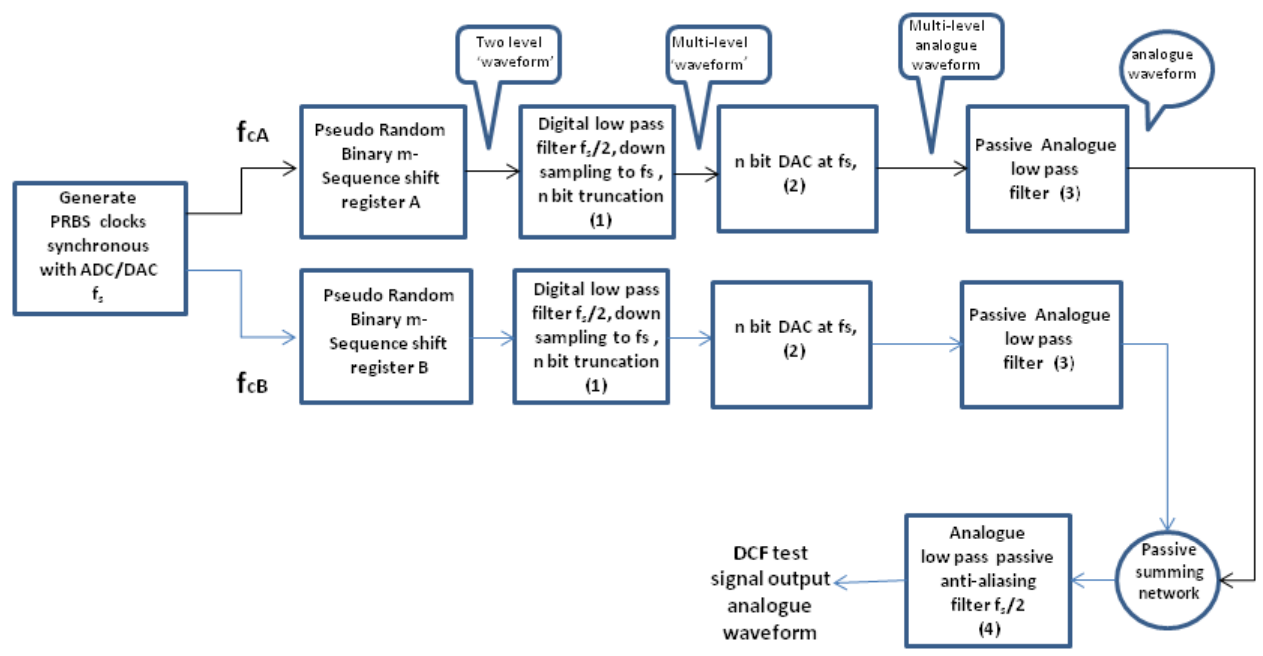

Fig. 2. Dual DAC-based synchronous DCF generator. Notes: the Nyquist sampling frequency is $f_{s}$ and block numbers are: (1) digital signal processing, (2) digital-to-analog converter, (3) analog LPF with bandwidth $f_{s} / 2$, and (4) optional antialiasing LPF with bandwidth $f_{s} / 2$.

that fall on the same spectral positions as the PRBS. This enables a reference amplitude pulse generator (or digital amplifier or any nonlinear amplifier) shown as (1) and (2) in Fig. 1 to be employed at that point. Noise in the amplifier or jitter on the clocks will produce an error that will appear between the spectral teeth of the test signal so must be minimized. LPFs (3) and (4) can be active if noise is low enough but passive if the output signal amplitude is sufficient. These filters convert the two level PRBS waveform into a smooth analog waveform with harmonics of equal amplitude. Passive summing ensures that this is not a source of nonlinearity. Finally, if the ADC under test does not include an antialiasing filter, a passive LPF (5) removes harmonics of the test signal above the ADC half sampling frequency. We will next consider the design choices to be made with the dual multibit DAC solution.

Fig. 2 depicts a practical DCF test signal generator using two DACs that operate at digital audio clock frequencies, for example $48 \mathrm{kHz}$. This is typical of DACs intended for stereo audio reproduction. However, as described in Section II, superior performance can be obtained if each DAC, (2) can be operated at sample rates harmonically related to the PRBS base frequency. Signal processing block (1) starts with a digital LPF operating at the common clock rate of $11232 \mathrm{kHz}$. The low-pass cutoff frequency of this filter is half the ADC sampling rate and provides an output word length that must be truncated to the word length of the DAC. Digital audio systems employ 24-bit DACs but these do not operate at a clock rate of $11232 \mathrm{kHz}$. It is therefore necessary to complete block (1) by subsampling the digital filter output to a frequency suitable for the DAC. Stopband attenuation of this filter is a critical consideration. When the filter output is down sampled to $48 \mathrm{kHz}$ any PRBS harmonics in the stopband will be aliased and appear as unwanted signals between the teeth of the test signal and limit the measurement precision.

As explained in Section II, in principle, carefully chosen PRBS lengths that have integer submultiples close to 48 $\mathrm{KHz}$ can avoid this aliasing problem but will require each
DAC to operate independently and at nonstandard sampling rates.

a) Selection of generator polynomials: One of the main challenges in designing the test signal generator is to select two PRBS base frequencies that minimize the number of harmonics that are common to both spectra. As the BBC work arrived at test signal base frequencies that achieved this aim, it was decided to choose frequencies close to these and implement the same PRBS generator polynomials [26], [27].

The $m$-sequence generator polynomials are $G_{12}(X)$ and $G_{11}(X)$

$$
\begin{aligned}
& G_{12}(X)=X^{12}+X^{11}+X^{8}+X^{6}+1 \\
& G_{11}(X)=X^{11}+X^{9}+X^{8}+X^{4}+1 .
\end{aligned}
$$

Referring to Fig. 2, an ADC/DAC sampling rate $f_{s}$ of $48 \mathrm{kHz}$ was selected with a passband of $20 \mathrm{kHz}$. Base frequencies of 152.4 and $109.7 \mathrm{~Hz}$ approximately were selected for the PRBS generators. These figures are close to those used by the BBC [26] but were chosen so that they were synchronous with $48 \mathrm{kHz}$ but not exact integer submultiples. This provided more than 300 spectral lines and ensured that most of the quantizing error and intermodulation products fell between the test signal components as harmonics are hidden by the test signal and have been predicted to be at least two orders of magnitude less in power [30]. A master clock frequency of $11252 \mathrm{kHz}$ was selected (234 times $48 \mathrm{kHz}$ ) for both test signal generator and DCF measurement system. This was divided by 36 to clock the 11-bit PRBS and divided by 25 to clock the 12-bit PRBS. If the 12-bit PRBS clock divider ratio is changed from 25 to 24 this generates a base frequency of approximately $114 \mathrm{~Hz}$. As $48 \mathrm{kHz}$ is the 420th harmonic of this base frequency this satisfies the test condition described in Section II and causes all nonlinearity products to be hidden by the test signal.

Simulations revealed that the minimum stopband attenuation of each LPF should be at least $160 \mathrm{~dB}$. This ensured that PRBS aliased harmonics are at least $20 \mathrm{~dB}$ below the expected level of 24-bit quantizing error. 
b) Selection of PRBS base frequencies and multibit DAC sampling frequencies to hide nonlinearity products: The aim is to select PRBS base frequencies and DAC sampling frequencies that cause all aliased products of nonlinearity to appear on harmonics of the PRBS base frequency. As has been mentioned in Section II, a Fourier series can generate a digital version of a multifrequency waveform where all components are harmonics of a common base frequency. We will refer to this as a comb spectrum with a sampling frequency $f_{\text {dac }}$. If the DAC sampling is $f_{\text {dac }}$ and comb base frequency $f_{\text {comb }}$ are harmonically related then nonlinearity products are aliased to the same frequencies as the test signal so are hidden. The following defines this condition in more detail.

The harmonic relationship required is

$$
f_{\text {dac }}=2 \cdot N \cdot f_{\text {comb }}
$$

where $N$ is the number of spectral lines up to and including $f_{\mathrm{dac}} / 2$.

Consider the $i$ th spectral line within the baseband frequency range up to $f_{\text {dac }} / 2$, its frequency $f_{i}$ is $i \cdot f_{\text {comb }}$ where $N \leq i>0$.

Next consider the $r$ th harmonic product $r_{h}$ of this $i$ th spectral line

$$
r_{h}=r \cdot i \cdot f_{\text {comb }}
$$

if $r \cdot i>N$ then $r_{h}$ becomes aliased to $f_{\text {dac }}-r_{h}$.

Substituting (13) and (14), we have the aliased values of $r_{h}$

$$
=2 \cdot N \cdot f_{\mathrm{comb}}-r \cdot i \cdot f_{\mathrm{comb}}=(2 N-r \cdot i) f_{\mathrm{comb}}
$$

as $N, r$, and $i$ are all integer the result is an integer so aliased $r_{h}$ falls on the in-band spectral lines of the test signal.

Next consider the general case where there is a frequency difference $\Delta f$ between the $N$ th spectral line and half sampling frequency

$$
0.5 f_{\mathrm{dac}}=\Delta f+N \cdot f_{\mathrm{comb}}
$$

the first harmonic $r_{\mathrm{h} \Delta}$ to occur above half sampling frequency is therefore $r_{h \Delta}=f_{\text {comb }}+N \cdot f_{\text {comb }}$ and this becomes aliased to

$$
\begin{aligned}
f_{\mathrm{dac}}-r_{h \Delta} & =2 \cdot\left(\Delta f+N \cdot f_{\mathrm{comb}}\right)-\left(f_{\mathrm{comb}}+N \cdot f_{\mathrm{comb}}\right) \\
& =2 \cdot \Delta f+(N-1) \cdot f_{\text {comb }} .
\end{aligned}
$$

In order for this result to be integer, $\Delta f$ must be 0 or a multiple of $0.5 f_{\text {comb }}$ otherwise the aliased harmonics will fall back into positions between the teeth of the comb.

We have therefore identified three conditions which result in the comb spectrum hiding aliased nonlinearity products

$$
\begin{aligned}
& f_{\text {dac }}=2 \cdot N \cdot f_{\text {comb }} ; \quad f_{\text {dac }}=(N-1) f_{\text {comb }} \\
& f_{\text {dac }}=N \cdot f_{\text {comb }} .
\end{aligned}
$$

Although a comb spectrum can be generated with a Fourier series in an arbitrary waveform generator it is easier to generate it using an $m$ stage linear shift register with feedback. This produces a PRBS. With a shift register clock frequency of $f_{\text {dac }}$ a comb spectrum is generate with the following relationship:

$$
f_{\mathrm{dac}}=f_{\mathrm{comb}}\left(2^{m}-1\right) \text {. }
$$

As (19) meets the conditions in (18) it follows that harmonic and intermodulation products are aliased back on to the comb spectrum so are hidden.

If this waveform is sub-sampled to a new frequency $f_{s}$ then comb frequencies that occur above $f_{s} / 2$ will be aliased. If there are $M$ spectral lines below $f_{s} / 2$ and the $M$ th is $\Delta f$ below $f_{s} / 2$ then the first spectral line above $f_{s} / 2$ will be aliased to $2 \cdot \Delta f+(M-1) \cdot f_{\text {comb }}$. Hence, for these products to be hidden, $\Delta f$ must be 0 or a multiple of $0.5 f_{\text {comb }}$.

If $\Delta f=0$ then $f_{s} / 2=(M-1) \cdot f_{\text {comb }}$ and as $f_{\text {comb }}=f_{\mathrm{dac}} /\left(2^{m}-1\right)$

$f_{s}=f_{\mathrm{dac}} 2 \cdot(M-1) /\left(2^{m}-1\right)$ or $f_{\mathrm{dac}} 2 \cdot M /\left(2^{m}-1\right)$.

For simplicity, $f_{s}$ should be generated by dividing $f_{\text {dac }}$ by an integer value. This restricts division ratios to those where $M$ and $\left(2^{m}-1\right)$ have common factors. However, other design options that give more flexibility in choosing $f_{s}$ are available and are presented in the following design example. The details in Fig. 2 are the basis for the following example.

c) Optimum choice of PRBS base frequencies: It is required to generate a PRBS waveform with a frequency spacing that can be reproduced in a comb filter. It is therefore necessary to have the clock rate of the comb filter synchronous with the PRBS base frequency. As the ADC sampling rate is $48 \mathrm{kHz}$, it follows that the comb filter clock rate must be a multiple of this. In this example, we choose this multiple to be 234. From (20), it follows that the PRBS clock rate must be an integer submultiple of $234 \times 48 \mathrm{kHz}$. We have selected a clock rate of $(234 \times 48) / 25 \mathrm{kHz}$ for the 12-bit PRBS and $(234 \times 48) / 36 \mathrm{kHz}$ for the 11-bit PRBS. The comb spacings are therefore

$$
\begin{aligned}
& \text { Comb1 }=((234 \times 48) / 25) /\left(2^{12}-1\right) \\
& \text { Comb2 }=((234 \times 48) / 36) /\left(2^{11}-1\right) .
\end{aligned}
$$

To hide all nonlinearity products, we need a DAC clock frequency that is close to $48 \mathrm{kHz}$ but is also a harmonic of the comb frequency. We therefore need to find the nearest integer to $N$ where $N$. Comb1 $\approx 48 \mathrm{kHz}$, so rearranging (21) we have $48 \approx N \cdot((234 \times 48) / 25) /\left(2^{12}-1\right)$ or $N \approx(4095 \cdot 25) /$ $234=437.5$ so $N=437$ or $N=438$ are solutions.

Taking $N=438$, the DAC clock frequency required for Comb1is $438((234 \times 48) / 25) /(4095) \approx 48.054 \mathrm{kHz}$.

For Comb2, $48 \approx N \cdot((234 \times 48) / 36) /\left(2^{11}-1\right)$ or $N \approx$ (2047.36) $/ 234=314.92$ so $N=315$ is a solution.

Taking $N=315$, the DAC clock frequency required for Comb2 is $315((234 \times 48) / 36) /(2047) \approx 48.011 \mathrm{kHz}$.

As the comb spectrum generated by each PRBS includes a component at the required DAC clock frequency all that is required is a digital phase-locked loop to select this frequency. As an alternative, if the PRBS length can be factorized into prime numbers a higher DAC clock frequency will avoid the need for a phase-locked loop.

For example, $4095=3^{2} \cdot 5 \cdot 7 \cdot 13$ and the nearest number greater than 438 that uses a selection of these factors is $455=5 \cdot 7 \cdot 13$, and hence, the DAC clock frequency required is $((48 \times 234) / 25)) / 9=49.92 \mathrm{kHz}$

This option is not available for comb2 as 2047 is prime so we must instead use a DAC clock frequency of 


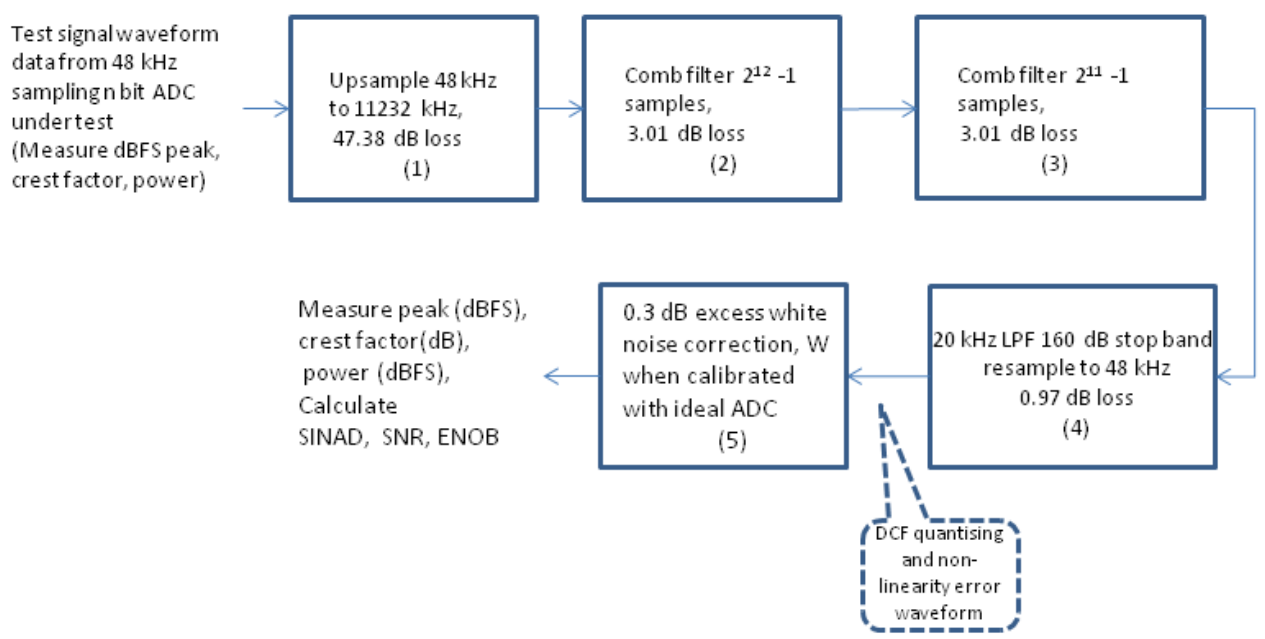

Fig. 3. DCF signal processing required to measure ENOB including specifications for each element of the measurement filter. Notes: block diagram numbers (1)-(4) comprise the signal processing elements of the measurement filter required to produce the nonlinearity error waveform and include the calculated white noise loss of each element on its own. (5) Value for white noise correction factor $W$ was estimated to be $0.3 \mathrm{~dB}$ (see $W_{M}$ in Fig. 5 ). $W$ is required to compensate for the fact that elements (3) and (4) do not have a white noise input.

$(48 \times 234 / 36)=312 \mathrm{kHz}$ or select a different shift register clock frequency and sequence length for comb2 that results in a lower DAC sampling frequency. We next consider the problem of generating a comb spectrum that is harmonically related to $48 \mathrm{kHz}$. This can be achieved simply by changing the division ration for Comb1 from 25 to 24 . We then have

Comb3 $=((234 \times 48) / 24) /\left(2^{12}-1\right)=48 / 420 \approx 114 \mathrm{~Hz}$.

As $420 \cdot$ Comb3 $=48 \mathrm{kHz}$, we have satisfied the requirements of (20) so all nonlinearity products of both DAC, amplifiers and ADC will be hidden by the test signal. This is a very powerful test as it provides a measure of interference and noise only. It will indicate if the system ENOB is dominated by noise and interference or nonlinearity.

2) Signal Processing and Measurement Filter Specifications for DCF Measurements: Fig. 3 depicts the individual filters and signal processing elements that comprise the measurement filter and also the measurement parameters required to calculate SINAD, SNR, and ENOB. The spectrum of each PRBS signal is removed by filtering with a comb filter rather than with complex high-order notch filters. This comb filter requires only a delay and subtractor so is easy to implement. However, comb filters do not have a uniform passband response so some quantizing error power is lost. An estimate of this white noise loss can be made by assuming that the comb filters have white noise as their input signal. White noise loss (W) for a digital filter with a maximum passband gain of unity is defined as follows.

1) $P_{i}$ is the mean squared amplitude of an input white noise signal of Nyquist bandwidth $B_{i}$.

2) $P_{o}$ is the mean squared amplitude of the output signal in a filter of Nyquist bandwidth $B_{o}$.

The white noise loss of this filter is defined only when $B_{o}=B_{i}$ to be

$$
W=P_{o} / P_{i}
$$

$W$ is determined by generating a white noise waveform of bandwidth $B$ and applying it to a digital filter of bandwidth $B$. White noise loss is the mean-squared amplitude of outputfiltered noise divided by the mean-squared amplitude of input white noise. In the particular case of a comb filter the white noise loss can be calculated as follows. Each comb filter has a transfer function of $1-z^{-m}$ where $z^{-1}$ is one sample period delay $T$ of $f_{\text {dac }}$ i.e., $T=1 / f_{\text {dac }}$ and $m$ is the length of its complimentary PRBS. The maximum gain of each comb filter must be $0 \mathrm{~dB}$ so the actual transfer function of the comb filter is $0.5\left(1-z^{-m}\right)$.

The magnitude-frequency response of a comb filter can be found by substituting $z=e^{j \omega}$.

This gives the result $H\left(\mathrm{e}^{j \omega}\right)=1-e^{-j(\omega \mathrm{mT})}$ and using Eulers formula, we find that the frequency response is $H\left(e^{j \omega}\right)=[1-\cos (\omega \mathrm{mT})]+j \sin (\omega \mathrm{mT})$, and hence the magnitude response is $|H(\omega)|=\sqrt{ }(2+2 \cos (\omega \mathrm{mT}))$ substituting $\omega=2 \pi f|H(f)|=2|\cos (\pi \mathrm{fmT})|$ and when adjusted to a maximum gain of 1 or $0 \mathrm{~dB}$ this becomes $\mid \cos (\pi \mathrm{fmT} \mid$.

To find the power loss/gain, we find its mean square value from the mean value theorem

$$
\begin{aligned}
\overline{|H(f)|^{2}} & =\frac{1}{2 \pi / \mathrm{mT}} \int_{-\pi / \mathrm{mT}}^{\pi / \mathrm{mT}}(|\cos (\pi \mathrm{fmT})|)^{2} d f \\
& =\frac{\mathrm{mT}}{2 \pi} \int_{-\pi / \mathrm{mT}}^{\pi / \mathrm{mT}}|\cos (\pi \mathrm{fmT})|^{2} d f=\frac{\mathrm{mT}}{2 \pi} \cdot \frac{\pi}{\mathrm{mT}}=0.5 .
\end{aligned}
$$

As $W=0.5$, in decibels this is $10 \log _{10}(0.5)=-3.01 \mathrm{~dB}$. Clearly, filter stopband attenuation is an important limitation to DCF maximum precision but in practice this difficulty can be overcome by offline filtering and storage. The generated waveform file can be replayed and then captured for off line processing. Conventional finite-impulse response (FIR) filter design software provided 160-dB stopband attenuation with an FIR of order 13352. Comb filters in Fig. 3 had delays of 102375 and 73692 samples at $11232 \mathrm{kHz}$. This ensured that they were perfectly aligned with the test signal spectrum. 
The generator and measurement system clocks must be locked to each other or a common reference. The measurement filter is actually a chain of filters: an up-down sampling rate converter and two comb filters. The sampling rate converter increases the sampling frequency from the $\mathrm{ADC}$ rate of $f_{s}$ to the signal generator DAC rate of $f_{\text {dac }}$. Sampling rate up-conversion is achieved by inserting $\left(f_{\text {dac }} / f_{s}\right)-1$ zero values after each sample at $f_{s}$. This results in the total baseband signal power reducing by $1 / R$, where $R$ is oversampling ratio $f_{\text {dac }} / f_{s}$. To change the sampling frequency from $f_{\text {dac }}$ to $f_{s}$ it is necessary to LPF the ADC data at $f_{\text {dac }}$ and select only one sample every $\left(f_{\text {dac }} / f_{s}\right)-1$ samples. This LPF is defined by its transfer function $H(z)$. It has a unity gain passband and $160-\mathrm{dB}$ stopband attenuation to prevent aliased energy above $f_{s} / 2$ from adding too much to the original ADC signal. Sampling rate conversion is a well-known DSP problem. Practical sampling rate converters often use multirate filtering achieve the sampling rate reduction in several stages. This results in each filter having less demanding stopband attenuation requirements than one filter alone.

If the length of each sequence is $p$ and $r$ samples then the transfer function of the comb filters is $\left(1-z^{-p}\right)\left(1-z^{-r}\right)$ then the total transfer function $Y(z)$ of the measurement filter (for white noise) is

$$
Y(z)=\left(1-z^{-p}\right)\left(1-z^{-r}\right) H(z) K,
$$

where

$K \quad$ white noise loss correction factor $K=k \cdot R$ / $\left(k_{p} \cdot k_{r} \cdot k_{H}\right)$

$k_{p} \quad$ white noise loss of the first comb filter;

$k_{r} \quad$ white noise loss the second comb filter;

$k_{H} \quad$ white noise loss the LPF;

$k \quad$ correction factor required to produce a unity white noise loss for $Y(z)$.

ADC quantizing noise has an aliased white spectrum. To measure its correct mean-squared value the measurement filter chain must have $0-\mathrm{dB}$ white noise loss. The white noise loss of each individual filter can be calculated and a first estimate of the total white noise loss can be obtained from the product of these. Clearly, this is not an accurate estimate as only the first filter in the chain has a white noise input. Unity white noise loss for the complete measurement filter is obtained by including the correction factor $k$. This must be determined experimentally as $k_{H}$ will vary with the choice of $H(z)$. Fig. 3 signal processing blocks give the initial values of gain and loss as presented next.

The overall gain $G$ required to achieve $0-\mathrm{dB}$ white noise loss through the measurement filter is calculated as follows:

1) each comb filter loss is $20 \log _{10}\left(1 / k_{p}\right)=3.01 \mathrm{~dB}$;

2) power loss due to sampling rate conversion is $20 \log _{10} 234=47.38 \mathrm{~dB}$

3) LPF white noise loss is $20 \log _{10}\left(1 / k_{h}\right)$ and was measured to be $0.97 \mathrm{~dB}$ but note that this figure will vary with the actual LPF design.

The assumption that the overall white noise loss is the sum of each individual filters loss is an approximation that we account for with a correction factor $W_{M}$. Hence, the required gain correction factor $G$ in decibels is

$$
\begin{aligned}
G & =20 \log _{10}(1 / k)+3.01+3.01+47.38+0.97 \\
& =20 \log _{10}(1 / k)+54.37=W_{M}+54.37 .
\end{aligned}
$$

The correct value for $G$ is dependent on both the LPF $k_{h}$ and on $W_{M}$. $W_{M}$ can be found by applying true quantizing noise of known mean-squared amplitude to the measurement filter input and finding the value of $W_{M}$ that gives the correct value of quantizing noise at the output. As simulations include a fixed number of DAC generator bits and a fixed number of samples, the correct value for $W_{M}$ was determined by a simulation running over a range of ADC bits sufficient to so see the effect of these on $W_{M}$ and this paper is reported next.

\section{B. Simulations to Determine Correct Measurement Filter Gain Factor $G$}

The DCF test signal was applied to a noise free linear quantizer and with this input, the waveform at the output of the measurement filter had a crest factor of $11.5 \mathrm{~dB}$. The crest factor of a waveform can be altered by changing the phase relationship of each spectral component in a nonlinear fashion and/or by adding or subtracting other waveforms with the same spectral content. As the measurement filter has a nonlinear phase characteristic it is expected to change the crest factor of any waveform that passes through it. If, for some unexpected reason, the measurement filter did not remove all the components of the original test signal this could also change the crest factor. This possibility was checked in the simulation by by-passing the measurement filter and instead subtracting an ideal DCF test signal from its quantized version. Theory predicts the following:

1) ideal quantizing noise waveform should have a crest factor of $4.77 \mathrm{~dB}$ before sampling;

2) aliasing will produce a white spectrum after sampling but with an unknown crest factor.

The simulated ideal quantizing noise waveform after sampling had a white spectrum and the unknown crest factor was actually $4.77 \mathrm{~dB}$. As aliasing had not changed the crest factor of the quantizing noise it was then likely that the measurement filter alone was the cause of the increase in crest factor. The quantizing noise waveform was applied to the measurement filter as this would then show the filter's effect on its crest factor. This test confirmed that the measurement filter changed the crest factor of the error waveform from 4.77 to $11.5 \mathrm{~dB}$ which is equal to the value obtained with the DCF measurement. The increase in crest factor of the quantizing noise was therefore due solely to the action of the measurement filter. As the rms value of quantizing error can be predicted from theory, the gain of the measurement filter could be corrected to give the correct rms quantizing noise value.

Simulated ideal DACs and ideal ADCs might be expected to give an SQNR equal to that predicted by theory. However, the following sources of measurement error $P_{m}$ prevent this being achieved:

1) quantizing noise $Q_{24}$ of an ideal 24-bit DAC is added to the ideal ADC quantizing error;

2) - $\mathrm{A} d \mathrm{~B}$ full scale (FS) error in setting the test signal amplitude to full scale produces an error in SQNR; 
Sine wave SINAD $(6.02 n+1.76)$

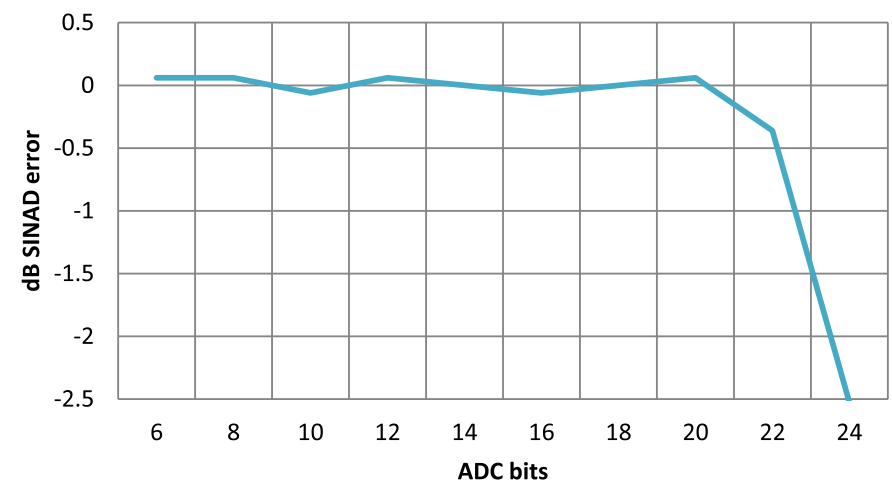

Fig. 4. Sine-wave SINAD measured with ideal ADC compared with theory based value for SINAD.

3) the limited number of samples in the test signal can result in missing codes and a measurement error $P_{n}$;

4) the value for $W_{M}$ may not be accurate.

Error sources (a)-(c) affect the measured value of SQNR for both DCF and sine-wave test methods. $Q_{24}$ adds to the ADC quantizing noise increasing the total by $3 \mathrm{~dB}$. This limits the 24-bit ADC measurement threshold for a sine wave to 23.5 bits. In contrast, as the DCF method requires summing two 24-bit DACs, this contributes $3 \mathrm{~dB}$ less quantizing noise, i.e., $Q_{24.5}$. This will increase the expected value for ideal 24-bit ADC quantizing noise by $1.7 \mathrm{~dB}$, limiting the measurement threshold to 23.7 bits.

Error (b) in setting the test signal amplitude will affect both methods equally so must be measured and results adjusted to give a full-scale range SQNR. This is achieved simply by increasing the SINAD by this decibel error value. As error source (c) is dependent on the number of samples for both methods, a number of samples close to $2 \mathrm{~s}$ at $48 \mathrm{kHz}$ was chosen for both methods. This was to facilitate a comparison of the accuracy of both methods in a routine test application. The number of samples for the DCF method was 95000 but to avoid DFT leakage a whole number of cycles of (approximately) $997-\mathrm{Hz}$ sine wave was generated with 65536 samples.

In the comparison, an ideal ADC was simulated from 8 to 24 bits.

Using the definition of ENOB presented in Section II, (1) and (2), all sources of error should be included when calculating ENOB but as $W_{M}$ was unknown, the value of $G$ used for the simulations had this value set to zero. Fig. 4 presents the result for (9) and Fig. 5 the results for (8) and (9). The decibel SINAD error in Figs. 4 and 5 refers to the difference between measured and expected results based on (7) when SQNR is replaced by SINAD. Even though an ideal ADC is simulated, the result is a SINAD rather than SQNR as it includes possible measurement error or uncertainty $P_{m}$. Fig. 4 indicates that the test parameter values selected gave an error of less than $\pm 0.1 \mathrm{~dB}$ at 20 bits and below. Above this the results are believed to be in error partly because there were insufficient samples in the test waveforms but also

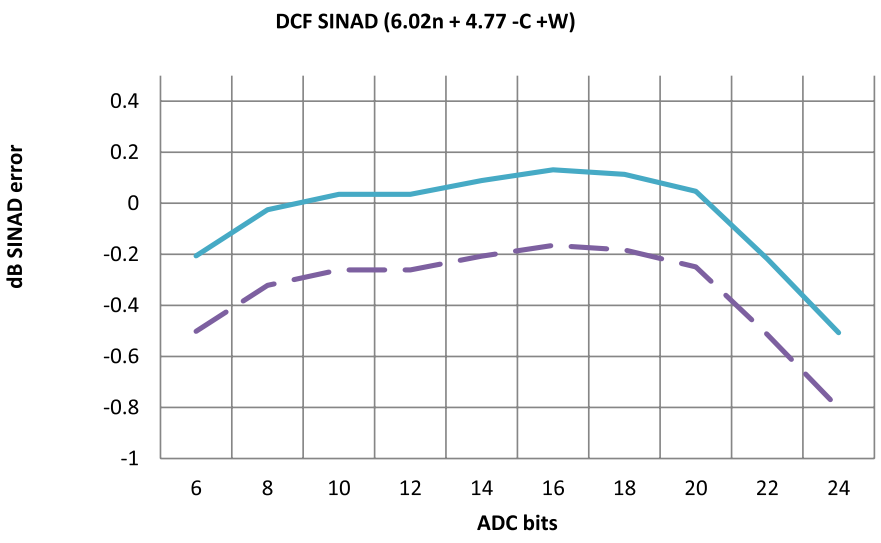

- error $\mathrm{dB} \longrightarrow .3 \mathrm{~dB}$ adjusted $\mathrm{dB}$ error

Fig. 5. DCF SINAD measured with ideal ADC compared with two theory based values for SINAD. Note: $W$ is the value of white noise loss correction factor. The dashed line applies to $W=0$ and the solid line applies to $W=W_{M}=0.3$, where $W_{M}$ is the value estimated to minimize the decibel SINAD error

because 24-bit DAC quantizing noise adds measurement error. Let $Q_{n}$ represent the ideal $n$-bit quantizing noise mean squared amplitude and $Q_{t}$ the test signal source quantizing noise mean squared amplitude. $M_{Q}$, the measured quantizing noise meansquared amplitude is given by

$$
M_{Q}=Q_{n}+Q_{t} .
$$

If $M_{T}$ is the measured test signal mean squared amplitude then the measured $n$-bit SINAD $S_{n}$ is

$$
S_{n}=M_{T} / M_{Q}
$$

and if $S_{n \mathrm{~dB}}$ is the SINAD in decibel then $S_{\mathrm{FSR} n \mathrm{~dB}}$ the SINAD corrected to full scale range (FSR) is

$$
S_{\mathrm{FSR} n \mathrm{~dB}}=S_{n \mathrm{~dB}}+A \mathrm{~dB} .
$$

The dashed line in Fig. 5 shows that the estimated white noise correction of $G=54.37 \mathrm{~dB}$ for the DCF measurement filter results in a SINAD error of $-0.3 \mathrm{~dB} \pm 0.2 \mathrm{~dB}$. This potential error $\left(W_{M}\right)$ in the estimate $G$ was highlighted previously as it takes into account the fact that the white noise loss approximation over estimates the loss in the complete filter chain. As the white noise loss of the LPF is design specific the value for $G$ will change and $W_{M}$ might also change with other LPF designs. Any new configuration of measurement filter must go through this simulated gain calibration procedure to determine the correct value of $G$. The reduction in SINAD error by including this systematic error $\left(W_{M}\right)$ as $-0.3 \mathrm{~dB}$ in all results is shown by the solid line in Fig. 5. This indicates the SINAD error for an ideal ADC is within $\pm 0.1 \mathrm{~dB}$ of the predicted value $n$ over the range $8-20$ bits

$$
\begin{aligned}
n \text { bit SINAD }(\mathrm{DCF}) \text { error }= & {\left[S_{n \mathrm{~dB}}(\mathrm{DCF})+A(\mathrm{DCF})-W_{M}\right] } \\
& -[6.02 n+4.77-C] \\
n \text { bit SINAD(Sine) error }= & {\left[S_{n \mathrm{~dB}}(\text { Sine })+A(\text { Sine })\right] } \\
& -[6.02 n+1.76] .
\end{aligned}
$$


A $0.3-\mathrm{dB}$ attenuation compensates for $W_{M}$ in the final block diagram of the DCF test system shown in Fig. 3. It is included in simulations and measurements presented in the following sections. In the following investigations using this measurement system, the total white noise correction gain $G$ is set to $54.07 \mathrm{~dB}$ instead of the estimated $54.37 \mathrm{~dB}$.

In the case of an ideal ADC, (9) should give an SQNR equal to that of a perfect ADC. This enables the precision of sine-wave- and DCF-based measurements to be compared directly. As $C$, the crest factor in $\mathrm{dB}$, is approximately $12 \mathrm{~dB}$ in the DCF test configuration described

$$
\mathrm{ENOB}=(\mathrm{SINAD}+7.23) / 6.02 \text { bits. }
$$

As the DCF SINAD is within $\pm 0.1 \mathrm{~dB}$ of the true value, from (28) this is an ENOB uncertainty of \pm 0.02 effective bits over the range $8-20$ bits. This is also within the range measured with a sine wave.

At the start of this section the option of using a single PRBS harmonically related to the ADC sampling frequency was discussed. If this configuration is used then all the quantizing error will appear on the PRBS spectral lines and be hidden. In that situation clearly (28) will not measure quantizing error. However, if noise is added to the ideal ADC then as random noise is not correlated with the test signal it is not hidden so (28) will give a noise-based ENOB. It will be demonstrated later that this feature can be used to determine the DCF SINAD and therefore ENOB when noise exceeds quantizing error. The practical details for this DCF test system were determined in the first part of EPSRC funded research project EP//D04264X/1. The next section presents simulations to evaluate the effect of added noise and nonlinearity on the comparative measurements of ENOB with sine and DCF methods. This is followed with results of measuring a professional quality audio 24-bit DAC-ADC unit.

\section{Simulations INCLUding Noise AND NONLINEARITY}

Section III has established an appropriate gain for the combfilter measurement system that provides the same value of $Q$ as a sine wave. The next stage in this paper is to determine the degree of agreement between sine and DCF measurements when Gaussian white noise is added to each test signal before it is quantized. The first simulation considers an ideal 24-bit ADC with white noise added at the same rms level as the ideal quantizing value $Q$.

\section{A. Ideal ADC}

Fig. 6 depicts the configuration used to evaluate the influence of additive white noise on the ENOB of three quantizers: an ideal 24 bit, practical 10 bit with a dominant low-order nonlinearity shown by the integral non linearity (INL) plot in Fig. 7 and a practical 8 bits with dominant segment boundary transition errors shown by the INL plot in Fig. 8 . For the practical 8- and 10-bit INL a white noise quantizing error was added at the $Q$ level of an ideal 8- and 10-bit ADC. The INL plots were measured using a servo loop method so the INL threshold values results are noise free. Adding noise makes the simulated ADC characteristic more realistic.

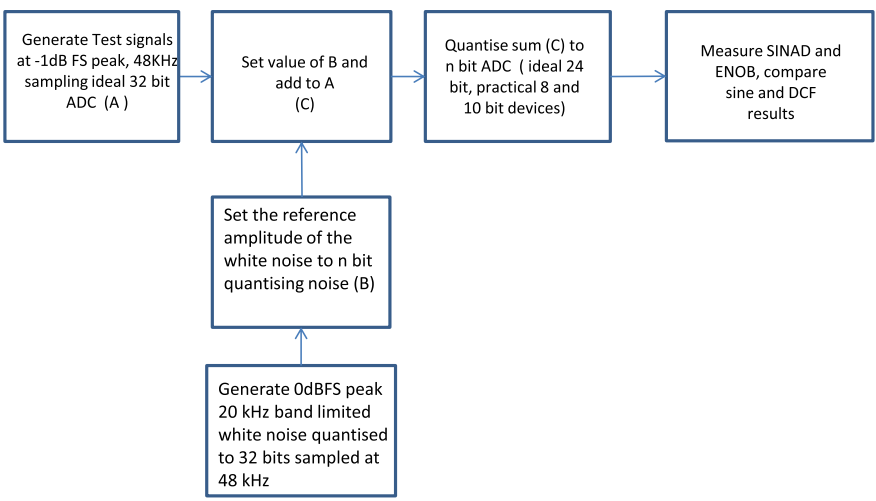

Fig. 6. Simulation block diagram for additive white noise for ideal 24-bit ADC.

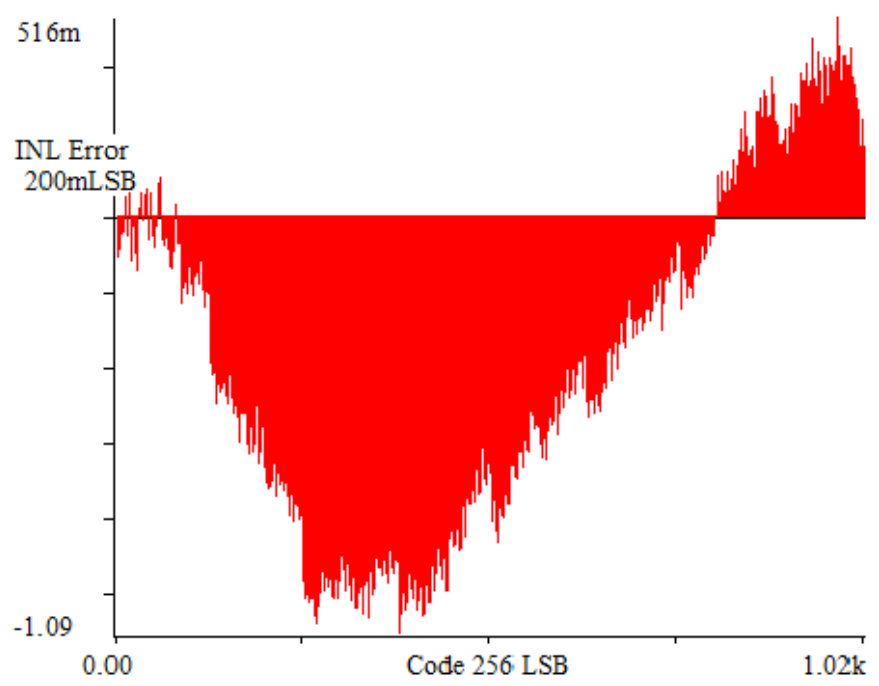

Fig. 7. 10-bit ADC INL.

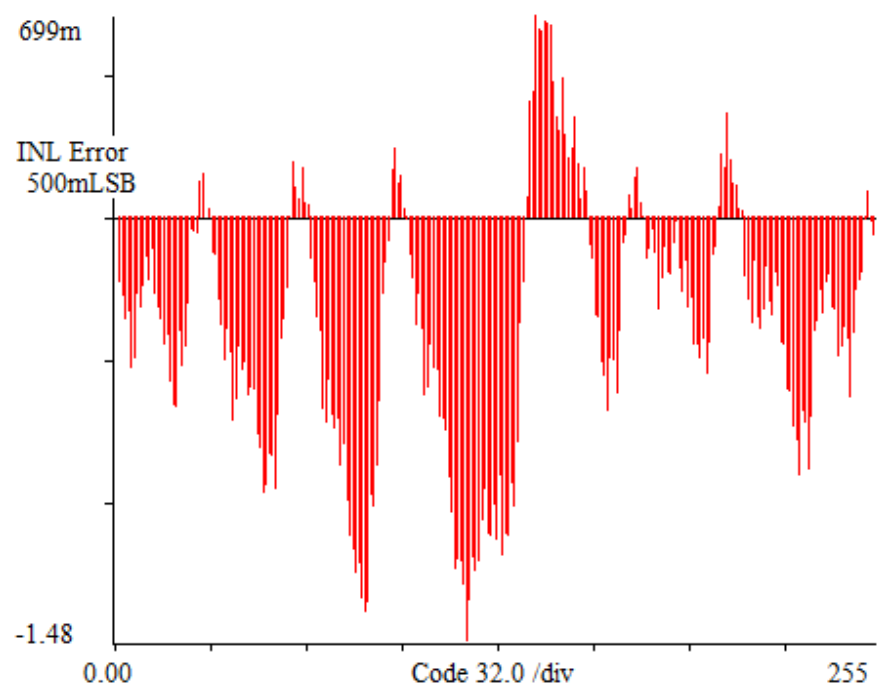

Fig. 8. 8-bit ADC INL.

Noise is added at an rms level equal to the rms quantizing error of an ideal ADC with the resolution under test and then in equal increments above this. Fig. 9 shows the simulated result of making these measurements with both a $997 \mathrm{~Hz}$ 
Sine and DCF ENOB at FSR ideal 24 bit ADC with Gaussian white noise added, 64k samples

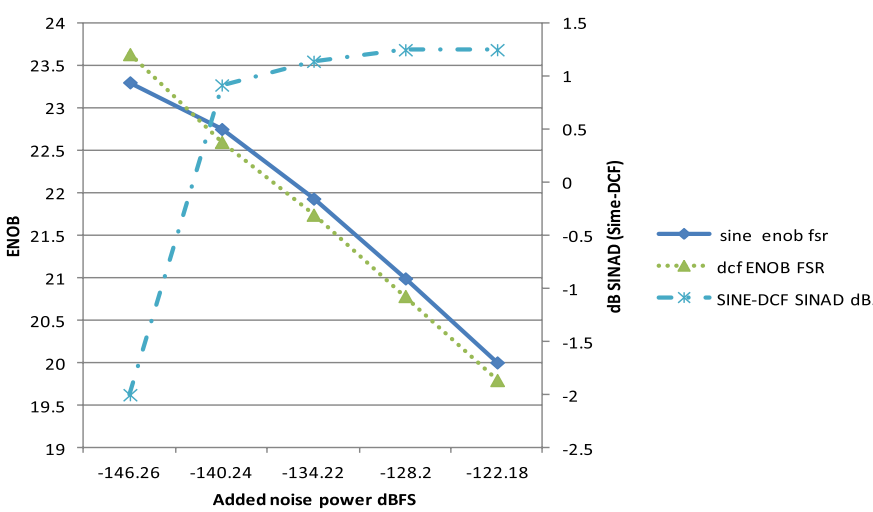

Fig. 9. Variation of sine and DCF ENOB with ideal ADC and added noise. Note: ENOB on the left axis applies only to the two plots with the square and triangle markers and is calculated from SINAD adjusted to full scale range. The decibel difference between SINAD measured with sine and SINAD measured with DCF applies only to the plot that has star markers.

sine synchronized to $48 \mathrm{kHz}$ and a DCF test signal with white noise added. The amplitude of this noise level is then increased in $6.02 \mathrm{~dB}$ increments until the total added Gaussian noise is $24.08 \mathrm{~dB}$ above the value of $Q$. This aspect of the simulation is intended to represent $P_{r}$ to find its the measurement uncertainty in $P_{m}$ (2).

At an added noise level of $Q(-146.26 \mathrm{~dB}$ FS $)$ the DCF method gives an ENOB close to the predicted value of 23.7 ENOB and then follows an almost linear progression in ENOB decrease with increasing added noise. Adding 4.5 bits of white noise to the ideal 24-bit ADC should reduces its ENOB from 24 to less than 20 bits. Each increment of $6.02 \mathrm{~dB}$ results in an increment in the ENOB closer to 1 bit and after 3.5 added bits both sine and DCF figures increase by the same amount but with an absolute difference of $1.3 \mathrm{~dB}$ or approximately 0.2 bits. In comparison the sine-wave ENOB has been reduced to 20 bits. One observation from the dashed plot with star markers is that the difference between sine and DCF ENOB measurements is greatest with an rms added noise value of $Q$ i.e., $-146.26 \mathrm{~dB}$ FS and the DCF method is better able to measure this. Increasing this by $6.02 \mathrm{~dB}$ is sufficient to make the two results follow a similar trend but 0.2 bits apart. A 64-k FFT was undertaken of the sine and PRBS test signal with 24-dB added noise. The PRBS test signal result is shown in Fig. 10 and the sine result in Fig. 11.

The net result is that the DCF method overvalues added band limited Gaussian white noise by about 0.2 bits when compared with a sine-wave measurement. A comparison of Figs. 10 and 11 noise floors shows that the spectral distributions are similar. Passband ripple in the DCF LPF is evident as is the fact that the Gaussian white noise is band limited to $20 \mathrm{kHz}$. If it can be first determined that the dominant source of error is band limited white noise then the DCF measurement filter gain can be increased to take this into account. The correction factor will depend on the bandwidth of the white noise but for the simulations presented here a value of $1.3 \mathrm{~dB}$ is estimated

$$
P_{r}(\text { sine })=P_{r}(\mathrm{DCF})-1.3 \mathrm{~dB} .
$$

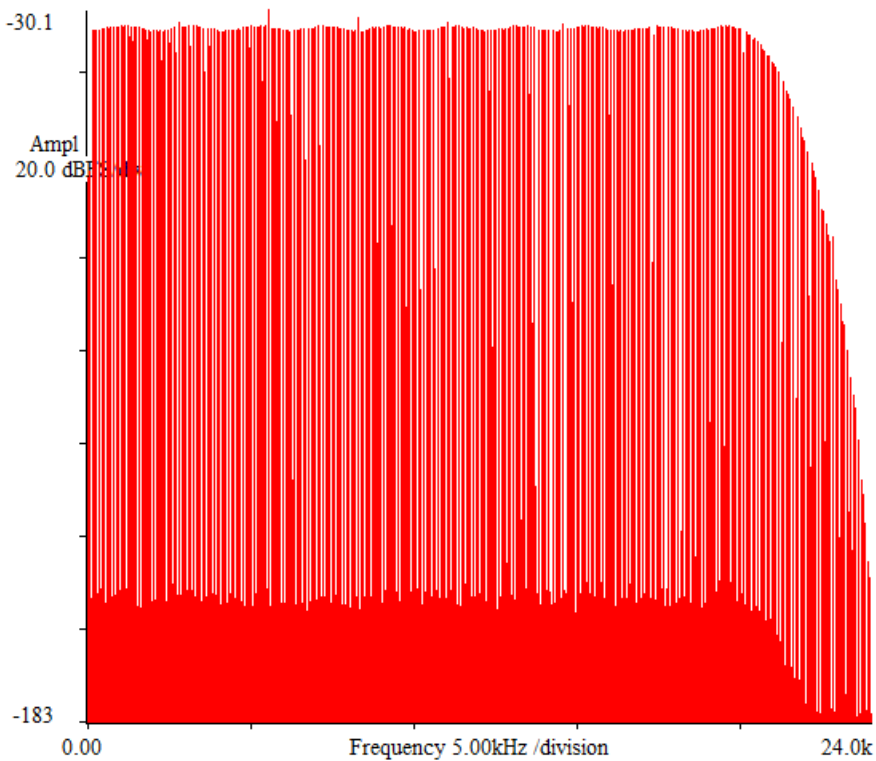

Fig. 10. Dual PRBS ideal 24-bit ADC with $Q+24 \mathrm{~dB}$ added noise.

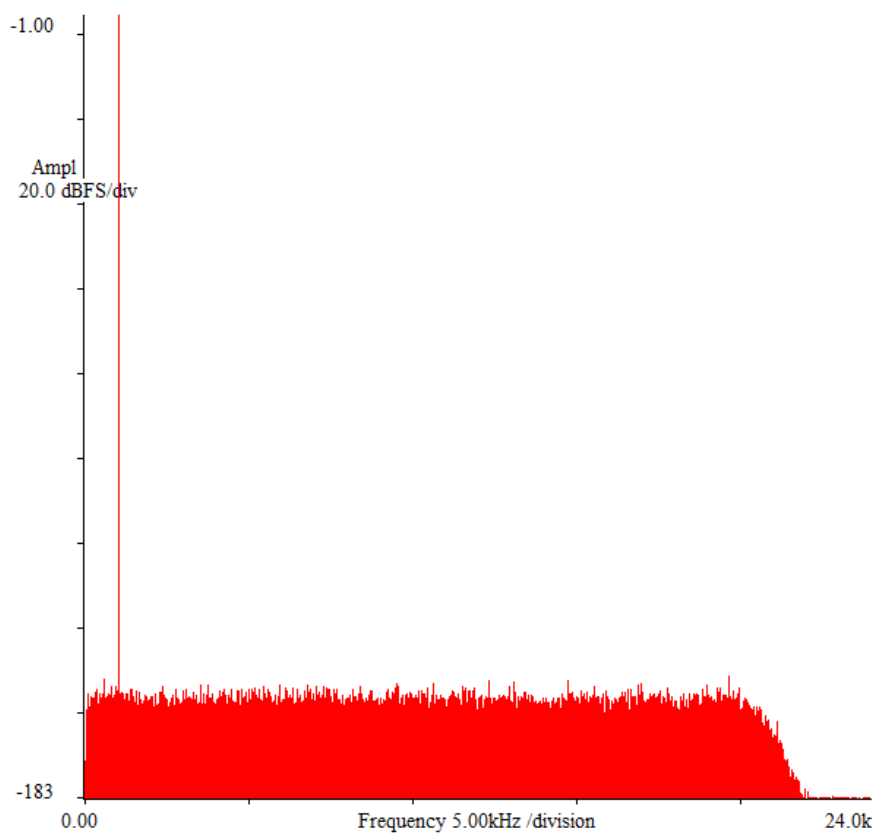

Fig. 11. 24-bit ideal ADC with $Q+24 \mathrm{~dB}$ added noise.

As Fig. 9 indicates that $P_{m}$ is a constant offset error when measuring $P_{r}$. This indicates that the measurement filter gain for random noise may be different to its gain for ideal quantizing error. This conclusion supports the simulation in Section III where a reduction in comb-filter gain $(0.3 \mathrm{~dB}$ after the LPF) was required to provide a more accurate estimate of ideal quantizing noise. Now the comb-filter gain needs to be increased by $1.3 \mathrm{~dB}$ when the sum of quantizing and added white noise results in white Gaussian white noise dominating the result. At this point it is important to remember that the present simulation uses band limited white noise but the Section III simulation used aliased quantizing noise. This could account for some of this $1.3 \mathrm{~dB}$ difference between 


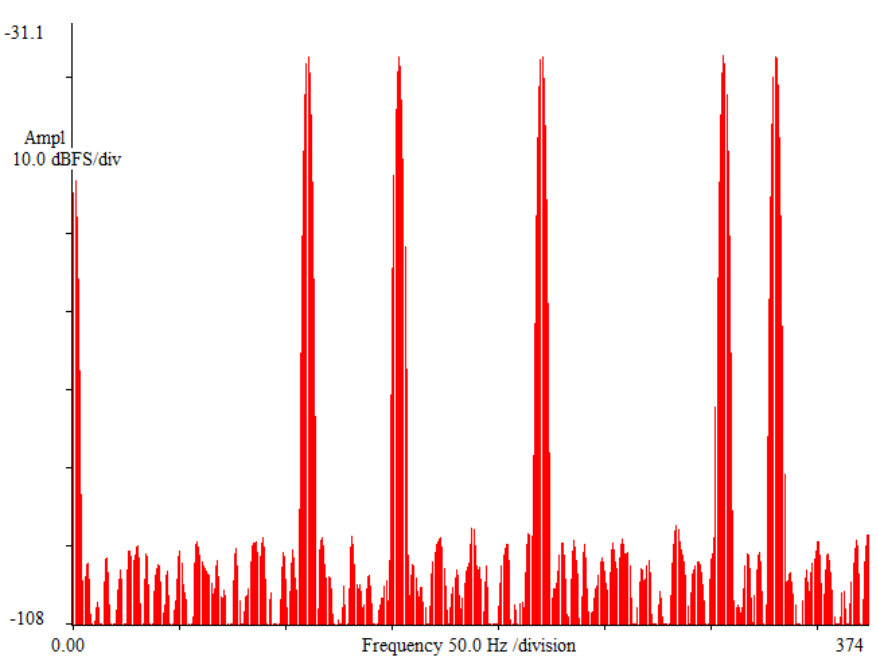

Fig. 12. 10-bit INL and one $Q$ noise DCF.

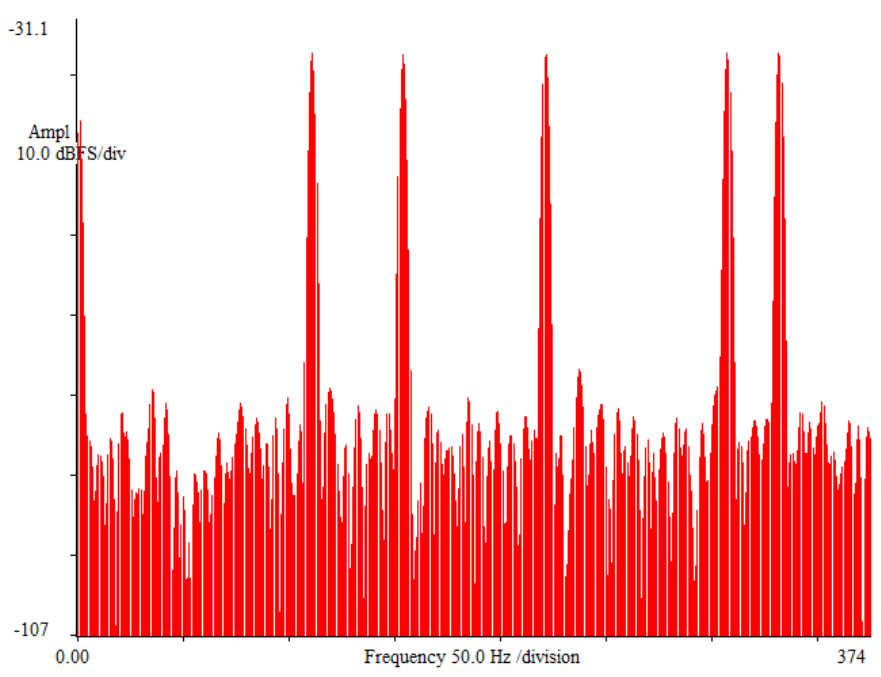

Fig. 13. 8-bit INL and one $Q$ noise.

the two simulations. In measurements of a practical ADC it will always be the case that sources of noise will be closer to Gaussian than uniform. Fortunately, using a single $114-\mathrm{Hz}$ PRBS will produce a result only if added random noise is present. The 1.3-dB correction factor to the DCF measurement filter could be applied if a 114-Hz PRBS measurement indicates that the ENOB is noise dominated.

\section{B. Simulation of Practical ADCs}

Fig. 12 shows the error spectrum between the teeth of the PRBS signal with the simulated 10-bit INL ADC and Fig. 13 gives the result for the 8-bit ADC. It is interesting to note that in each case the error has a line spectrum. The peaks in the error spectrum for the 8-bit INL are more noticeable.

The sine-wave FFT for the 10-bit ADC shown in Fig. 14 indicates that low-order nonlinearity is dominant. Fig. 15 shows that the segment transition errors in the 8-bit ADC generate high-order harmonics. In most applications of ADCs the spectrum in Fig. 14 is more likely to give acceptable wideband results than the spectrum in Fig. 15.

The results for the 8- and 10-bit ADCs with INL error and added noise of one $Q$ are presented in Table I and apply with

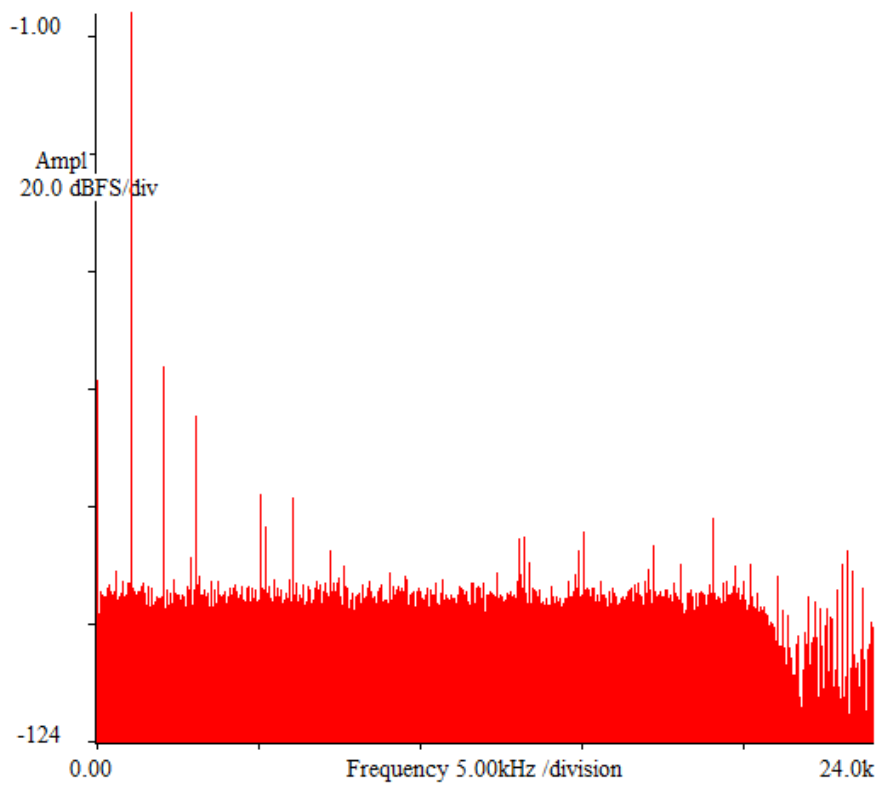

Fig. 14. 10-bit INL and one $Q$ noise.

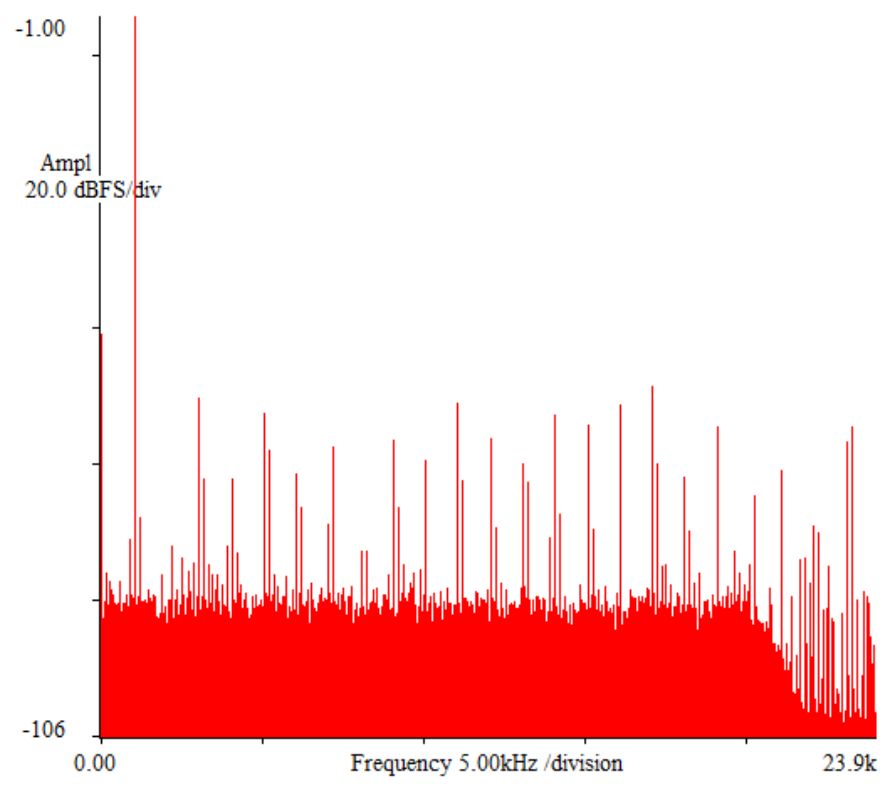

Fig. 15. 10-bit INL and one $Q$ white noise, full spectrum.

a sine-wave amplitude of $-1-\mathrm{dB}$ FS and DCF peak amplitude of $-1-\mathrm{dB}$ FS.

With the 8-bit ADC, the DCF method, the results in Table I show that it provides an ENOB of 0.47 bits less than a sine. SINAD measurements with the $114-\mathrm{Hz}$ PRBS indicated that the SINAD result was not noise dominated so the $1.3-\mathrm{dB}$ correction factor was not required for 8- or 10-bit ADCs. The DCF 10-bit result is 0.42 ENOB better than for a sine wave. It is concluded that the DCF ENOB figure gives a result that may be more useful for wideband applications as it enables high-order nonlinearity to be highlighted. Returning to the error model for ENOB, it has now been demonstrated that the DCF method provides a fundamentally different rms value for $P_{\mathrm{INL}}$ than a sine wave so there should be no expectation that this aspect of ENOB should agree in both methods. With regard to $P_{\mathrm{DNL}}$, it is possible that with the dual PRBS this 
TABLE I

Sine AND DCF Results For Simulated 8 AND 10-Bit ADCs With Noise

\begin{tabular}{|l|l|l|l|l|l|l|l|l|l|}
\hline ADC & $\begin{array}{l}\text { SINAD } \\
\mathrm{dB}\end{array}$ & THD dB & SNR dB & $\begin{array}{l}\text { Sine } \\
\text { ENOB }\end{array}$ & $\begin{array}{l}\text { PRBS } \\
\text { Power } \\
\text { dB FS }\end{array}$ & $\begin{array}{l}\text { Crest } \\
\text { factor } \\
\text { dB }\end{array}$ & $\begin{array}{l}\text { DCF } \\
\text { power } \\
\text { dB FS }\end{array}$ & $\begin{array}{l}\text { DCF } \\
\text { SINAD } \\
\text { dB }\end{array}$ & $\begin{array}{l}\text { DCF } \\
\text { ENOB }\end{array}$ \\
\hline $\begin{array}{l}8 \text { bit } \\
\text { INL }\end{array}$ & 44.43 & -52.46 & 45.06 & 7.07 & -10.03 & 12.08 & -42.43 & 32.4 & 6.6 \\
\hline $\begin{array}{l}10 \text { bit } \\
\text { INL }\end{array}$ & 56.77 & -58.98 & 60.77 & 9.14 & -9.98 & 11.98 & -60.36 & 50.38 & 9.56 \\
\hline
\end{tabular}

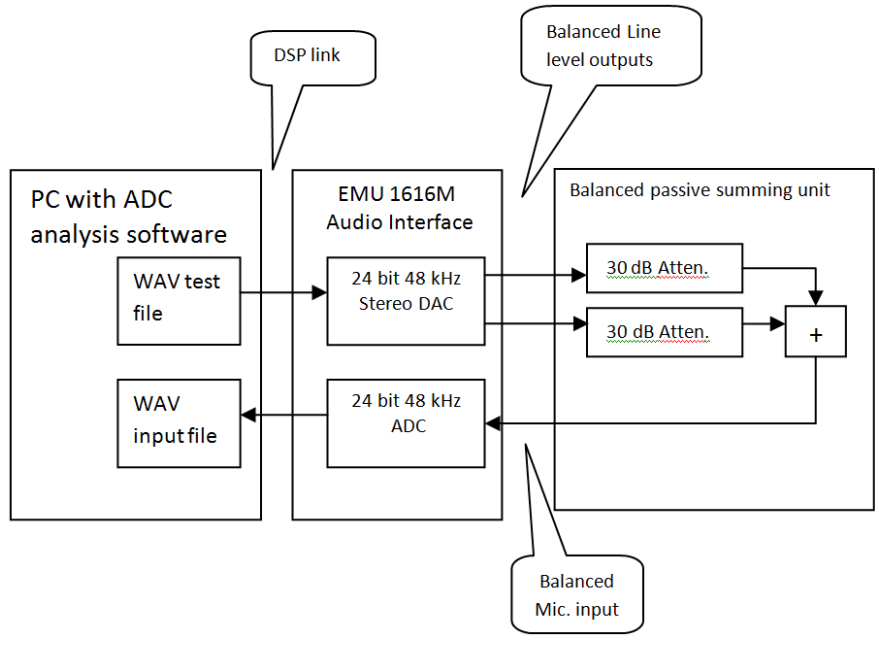

Fig. 16. 24-bit ADC DCF test block diagram.

adds to the total random error so the dual PRBS might provide $\left(P_{\mathrm{DNL}}+P_{r}\right)$ similar to a sine wave. If this sum generates noise then it can be measured using the 114-Hz PRBS.

As measurement filter correction gains have been determined for $Q$ and $\left(P_{\mathrm{DNL}}+P_{r}\right)$, only $P_{\mathrm{INL}}$ prevents ENOB for sine and DCF measurement methods agreeing. It is therefore proposed that the DCF method is able to make an accurate measurement of ENOB but should be used as a wideband ENOB. This ENOB figure is unlikely to agree with sine-wave ENOB unless the ADC ENOB figure is noise dominated.

The next section describes results obtained when these three measurements (sine, dual PRBS, and single PRBS) are applied to testing a real 24-bit audio ADC.

\section{Measurements With a Real 24-Bit ADC-DAC}

The practical test results reported in this section were undertaken using part of an experimental setup for measuring microphone nonlinearity. Fig. 16 shows the main aspect of the sine and DCF measurements. PC-based software generated 24bit $48-\mathrm{kHz}$.wav files for the $997-\mathrm{Hz}$ sine wave and the single and dual PRBS test signals. As two of the DACs were required to generate the DCF test signal the .wav file was set to stereo format. The E-MU Systems Inc (EMU) $1616 \mathrm{M}$ unit has a digital signal processor that enables .wav files to be replayed to generate the test signals and simultaneously captures .wav files from the ADC. ENOB was calculated by importing the .wav files into analysis software. Balanced outputs and inputs were employed to minimize the pick-up of power line interference. The two PRBS signals were summed in a balanced resistive network. The 30-dB attenuators provided isolation between each DAC and this minimized the possibility of nonlinear crosstalk degrading the results. Fortunately, the difference in amplitude between the line level outputs and the microphone input allowed this $30-\mathrm{dB}$ attenuation without degrading signal-to-noise ratio.

The results for the Creative Labs Professional Audio 24-bit audio ADC and DAC unit model EMU $1616 \mathrm{M}$ are presented next. Sine-wave measurements were made at $997 \mathrm{~Hz}$ with an FFT using 64-k samples and DCF measurements used 95000 samples. The DCF measurement results for the special case of one $114-\mathrm{Hz}$ PRBS, where the result automatically excludes amplitude nonlinearity, are shown in bold in Table II. They indicate that the DCF power is not dominated by nonlinearity. This can be concluded as the single PRBS DCF power is close to the power of the dual PRBS and also close to the SNR and idle noise in the sine-wave measurements. This situation where all three measurements have a similar noise figure highlights a further practical issue. Noise may be a spurious periodic signal that falls in the passband of the comb filters. The difference between DCF and sine ENOB is 0.83 ENOB and reflects simulation results where low-order nonlinearity generates a smaller rms error in the DCF result. As this is a practical test it reveals a further limitation of both sine and DCF measurements. Power line interference at $50 \mathrm{~Hz}$ and its harmonics appear and increase the noise floor below $2 \mathrm{kHz}$. A 70-Hz high-pass digital filter was therefore applied in the sine-wave and DCF measurements. Fig. 17 (right) shows that the $50-\mathrm{Hz}$ power line interference was at $-100-\mathrm{dB}$ FS as was the third harmonic of $997 \mathrm{~Hz}$. Fig. 17 (left) shows that a 70-Hz high-pass filter (HPF) reduces the power line harmonics to below 110-dB FS so they no longer dominate the SINAD figure. However, the power line harmonics are still more than $20 \mathrm{~dB}$ above the wideband noise level and degrade the SNR result. The SNR in the range $12-24 \mathrm{kHz}$ was calculated as this figure excluded harmonics and the power line interference. When corrected to full bandwidth and full-scale range the calculated SNR was $107.3 \mathrm{~dB}$. Table II shows that the SNR including residual power line harmonics was $103.35 \mathrm{~dB}$, and therefore, the power line harmonics have degraded the SNR by $4 \mathrm{~dB}$. Comparing Fig. 17 with Fig. 18, it is clear that both test methods have measured noise levels that are likely to be in error as they include power line harmonics.

Hence, noise dominates ENOB for the PRBS test and harmonic distortion dominates ENOB for the sine test. The ENOB at FSR was estimated assuming that noise dominated the result so a simple gain correction was included. However, this would undervalue the rms error that could be caused by nonlinearity and as the dual PRBS test signal peak is $1.4 \mathrm{~dB}$ less than the sine wave the ENOB may be too high. This highlights a further experimental issue in comparing sine 
TABLE II

ENOB MEASurements For 24-Bit Audio ADC

\begin{tabular}{|l|l|l|l|l|l|l|l|l|l|l|}
\hline $\begin{array}{l}\text { Sine } \\
\text { power } \\
\text { dB FS }\end{array}$ & $\begin{array}{l}\text { SINAD } \\
\text { dB }\end{array}$ & SNR dB & $\begin{array}{l}\text { ENOB } \\
\text { at FSR }\end{array}$ & $\begin{array}{l}\text { Idle } \\
\text { noise } \\
\text { dB FS }\end{array}$ & $\begin{array}{l}\text { PRBS } \\
\text { peak } \\
\text { dB FS }\end{array}$ & $\begin{array}{l}\text { PRBS } \\
\text { power } \\
\text { dB FS }\end{array}$ & $\begin{array}{l}\text { Crest } \\
\text { factor } \\
\text { dB }\end{array}$ & $\begin{array}{l}\text { DCF } \\
\text { power } \\
\text { dB FS }\end{array}$ & $\begin{array}{l}\text { DCF } \\
\text { SINAD } \\
\text { dB }\end{array}$ & $\begin{array}{l}\text { DCF } \\
\text { ENOB } \\
\text { aSR } \\
\text { FSR }\end{array}$ \\
\hline-1.41 & 97.19 & 102.34 & 16.09 & -103.35 & -2.8 & -11.89 & 12.03 & -103.69 & 91.8 & 16.92 \\
\hline & & & & & $-\mathbf{2}$ & $\mathbf{- 8 . 5}$ & $\mathbf{9 . 4 3}$ & $\mathbf{- 1 0 4}$ & $\mathbf{9 5 . 5}$ & $\mathbf{1 6 . 9 6}$ \\
\hline
\end{tabular}
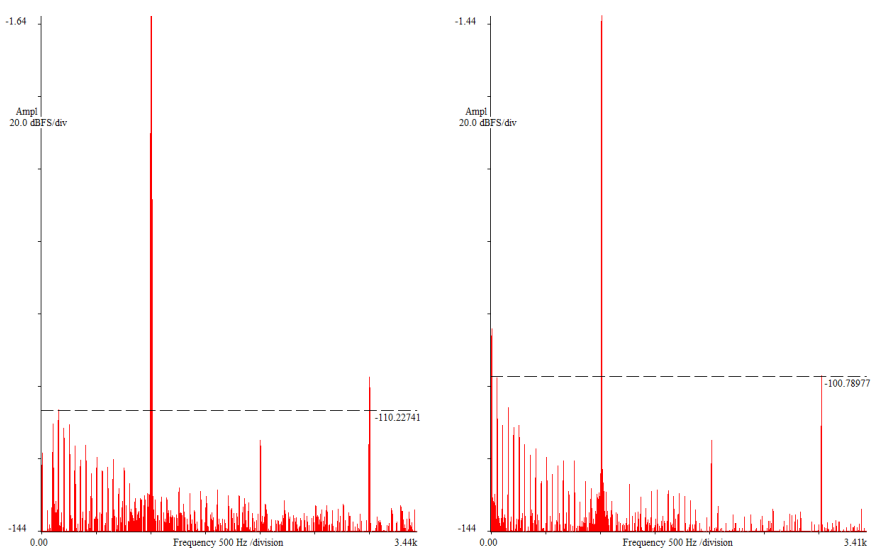

Fig. 17. EMU FFT (left) $50 \mathrm{~Hz}$ removed by $\mathrm{HPF}$ and (right) $50 \mathrm{~Hz}$ at $-100 \mathrm{~dB}$ FS without HPF.

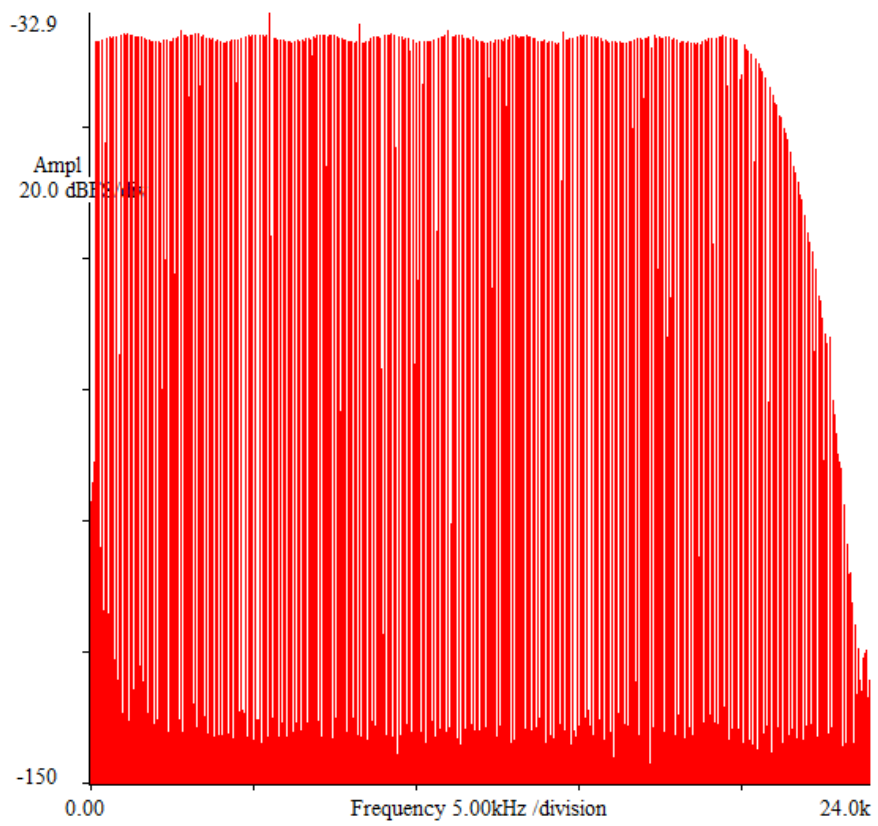

Fig. 18. DCF FFT no HPF.

and DCF ENOB. Ideally, the comparison should be made as in the simulations, with sine and PRBS at the same peak. The .wav files had been generated with PRBS and sine signals but the EMU requires separate gain adjustments to set signal amplitudes just below overload.

Fig. 19 shows the test setup in operation with the main components and interconnections. Ground connections between the PC and the audio unit were isolated by the digital RJ45 link between the PC interface card and the EMU unit. In conclusion, the practical results have revealed two aspects not included in the simulations: periodic interference and

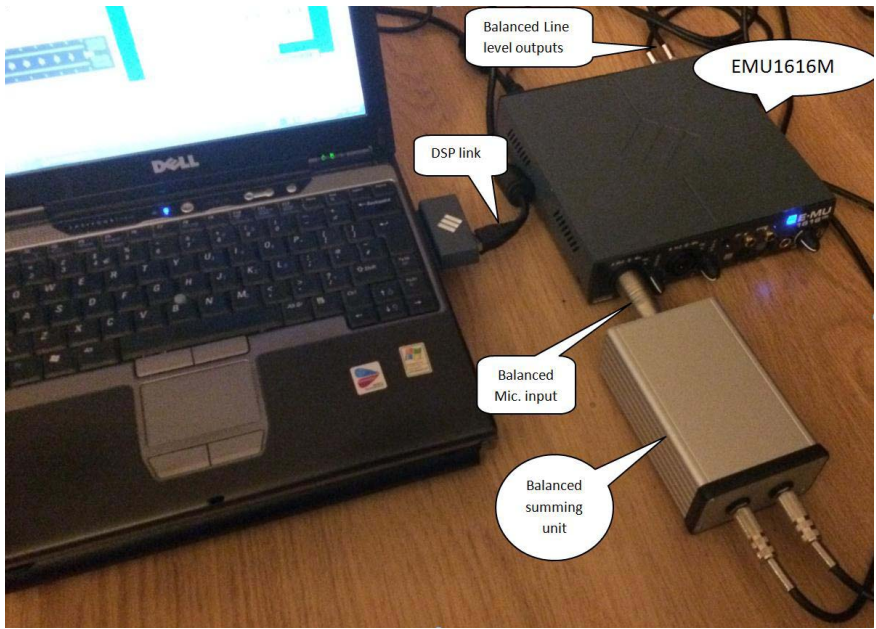

Fig. 19. DCF test setup practical 24-bit ADC testing.

the difficulty in setting peak amplitudes to be equal. The main difficulty in adjusting the signal levels to be close to each other was that the level meter in the EMU software responded correctly to a sine wave but under-valued the PRBS signal peaks. Wideband ENOB of a practical 24-bit ADC can therefore be measured using the PRBS test signals and results support those of simulations but include practical imperfections of a 24-bit DAC. The rms value of amplitude nonlinearity is weighted differently by each test signal and the results have demonstrated that this factor, rather than lack of precision in the DCF method, is the main reason why sine and DCF-based ENOB do not give the same figures. It is likely that, particularly for audio, RF, and microwave communication systems, the DCF-based ENOB is a better representation of system performance than a sine-wave ENOB.

\section{CONCLUSION}

This paper has shown that the DCF wideband multitone method specified in IEC standard 60748-4-3 can be better defined so that appropriate choices of test signal frequencies and measurement filter can be made. The SINAD or SQNR of an ideal ADC can be now measured with the DCF system within $0.1 \mathrm{~dB}$ of the SINAD or SQNR of an ideal sine wave. The IEC standard specifies the use of both a single PRBS and dual PRBS test signal. A particular form of single PRBS test signal is able to facilitate a measurement of SNR by excluding amplitude nonlinearity. The results in this paper have shown that this separate measurement enables the white noise gain of the DCF measurement filter to be adjusted to correct the ENOB figure for ADCs where the ENOB is dominated by white noise. It does not suffer from the curve-fitting problems 
associated with sine-wave measurements of ENOB. This is a potentially low-power method suitable for built in test or for self-test of programmable system on chip devices and ASICs that have ADCs and DACs available as IP blocks. The test will use less of the field-programmable gate array real estate than conventional methods such as FFT, curve-fitting, or histogram analysis. The results of simulations and practical measurements have shown that close agreement with the sinewave ENOB does not hold when amplitude nonlinearity is present.

A sine wave will be more sensitive to nonlinearity at the peaks so when this is dominant it may give a lower ENOB than the DCF method. Nonlinearity near the center of the transfer characteristic or where segment matching errors provide transitions or high-order nonlinearity is likely to give a lower ENOB with the DCF method than with a sine wave. In modern communication systems, an ENOB with this feature is likely to be more useful in indicating the suitability of the ADC as high-order harmonics generate a wide distortion spectrum and are more audible in the audio application than low-order harmonics. An ENOB based on the DCF test may therefore prove to be of more practical use as it is likely to be more representative of real world signals. This could avoid the potential problem of an ADC passing a sine-wave ENOB specification but failing to meet overall system requirements. It is recommended that wideband ENOB, based on the results presented here, should be included as an additional measurement parameter in any future revision of the IEC standard IEC 60748-4-3.

\section{REFERENCES}

[1] Semiconductor Devices-Integrated Circuits-Part 4-3: Interface Integrated Circuits-Dynamic Criteria for Analogue-Digital Converters (ADC), document IEC 60748-4-3:2006, Aug. 2006.

[2] IEEE Standard for Terminology and Test Methods for Analog-to-Digital Converters, IEEE Standard 1241-2010, Jan. 2011.

[3] ISO/IEC Directives Part 1 and Consolidated ISO Supplement, 5th ed., 2014.

[4] ISO/IEC Directives Part 2-Rules for the Structure and Drafting of International Standards, 6th ed., ISO/IEC DIR 2, 2011.

[5] S. Max and R. Liggiero, "Reducing THD in an audio test instrument," in Proc. IEEE Int. Instrum. Meas. Technol. Conf. (I2MTC), May 2012, pp. 2268-2273.

[6] Q-WAVE: A Quantum Standard for Sampled Electrical Measurements. [Online]. Available: http://www.ptb.de/emrp/qwave.html, accessed Aug. 20, 2013.

[7] L. Palafox, R. Behr, O. F. O. Kieler, and F.-J. Ahlers, "Ultrapure sinewave generation by combining Josephson systems," in Proc. 17th IMEKO, Barcelona, Spain, Jul. 2013, pp. 194-196.

[8] R. Behr, O. F. O. Kieler, D. Schleußner, L. Palafox, and F.-J. Ahlers, "Combining Josephson systems for spectrally pure AC waveforms with large amplitudes," IEEE Trans. Instrum. Meas., vol. 62, no. 6, pp. 1634-1639, Jun. 2013.

[9] S. Li et al., "A 12-pole narrowband highly selective high-temperature superconducting filter for the application in the third-generation wireless communications," IEEE Trans. Microw. Theory Techn., vol. 55, no. 4, pp. 754-759, Apr. 2007.

[10] J. C. Pedro and N. B. Carvalho, "Designing multisine excitations for nonlinear model testing," IEEE Trans. Microw. Theory Techn., vol. 53, no. 1, pp. 45-54, Jan. 2005.

[11] M. Friese, "OFDM signals with low crest-factor" in Proc. IEEE GLOBECOM, vol. 1. Nov. 1997, pp. 290-294.

[12] R. A. Belcher and J. A. Chambers, "Integral non-linearity error measurement through statistical analysis of a multi-tone test signal," in Proc. 7th IMA/IET Int. Conf. Math. Signal Process., Dec. 2006, pp. 1-4.
[13] J. Goes, N. Paulino, and M. D. Ortigueira, "Digital-domain selfcalibration technique for video-rate pipeline A/D converters using Gaussian white noise," Electron. Lett., vol. 38, no. 19, pp. 1100-1101, Sep. 2002.

[14] M. G. C. Flores, M. Negreiros, L. Carro, and A. A. Susin, "INL and DNL estimation based on noise for ADC test," IEEE Trans. Instrum. Meas., vol. 53, no. 5, pp. 1391-1395, Oct. 2004.

[15] S. Nikaido and T. Nitatori, "Non-linear distortion measurements of audio systems using noise signal," in Proc. 7th Int. Congr. Acoust., vol. 1. Budapest, Hungary, 1971, p. 301.

[16] S. Nikaido, "Dynamic measurement of non-linearity distortion on sound transmission systems," Eur. Broadcast. Union, Geneva Switzerland, Tech. Rep. 131, 1972

[17] F. H. Irons, K. J. Riley, D. M. Hummels, and G. A. Friel, "The noise power ratio-Theory and ADC testing," IEEE Trans. Instrum. Meas., vol. 49 , no. 3 , pp. $659-665$, Jun. 2000.

[18] J. Schoukens and T. Dobrowiecki, "Design of broadband excitation signals with a user imposed power spectrum and amplitude distribution," in Proc. IEEE IMTC, Saint Paul, MN, USA, May 1988, pp. 1002-1005.

[19] T. Jiang and Y. Wu, "An overview: Peak-to-average power ratio reduction techniques for OFDM signals," IEEE Trans. Broadcast., vol. 54, no. 2, pp. 257-268, Jun. 2008.

[20] G. Srinivasan et al., "Accurate measurement of multi-tone power ratio (MTPR) of ADSL devices using low cost testers," in Proc. IEEE Eur. Test Symp., May 2005, pp. 68-73.

[21] C. Huang, M. Zhang, J. Wu, S. Lu, and L. Shi, "On the use of multi-tone for the measurement of noise power ratio distortion in RF circuits," in Proc. IEEE Asia Pacific Conf. Circuits Syst., Nov./Dec. 2008, pp. $1596-1599$

[22] I. Kollar and J. J. Blair, "Improved determination of the best fitting sine wave in ADC testing," IEEE Trans. Instrum. Meas., vol. 54, no. 5 , pp. 1978-1983, Oct. 2005.

[23] W. R. Bennett, "Spectra of quantized signals," Bell Syst. Tech. J., vol. 27, no. 3, pp. 446-472, 1948.

[24] J. J. Blair, "Selecting test frequencies for sinewave tests of ADCs," in Proc. 19th IEEE IMTC, vol. 1. May 2002, pp. 189-193.

[25] A. Gharib, A. Talai, R. Weigel, and D. Kissinger, "A 1.16 pJ/bit 80 $\mathrm{Gb} / \mathrm{s} 2^{11}-1$ PRBS generator in SiGe bipolar technology," in Proc. 9th Eur. Microw. Integr. Circuit Conf. (EuMIC), 2014, pp. 277-280.

[26] R. A. Belcher, "Audio non-linearity: An initial appraisal of a double comb-filter method of measurement," British Broadcast. Corp. Kingswood, U.K., Res. Rep. 1977/40, Jan. 1977.

[27] R. A. Belcher, "Test signals for the measurement of non-linear distortion of sound signals," Ph.D. dissertation, Dept. Electron. Eng., Univ. Surrey, Guildford, U.K., Jun. 1977, p. 81.

[28] R. A. Belcher, "A double comb filter method for testing of quantisers," in Proc. IEE 3rd Int. Conf. Adv. A/D D/A Covers. Tech. (ADDA), Jul. 1999, pp. $79-82$.

[29] S. W. Golomb and G. Gong, Signal Design for Good Correlation: For Wireless Communication, Cryptography, and Radar. Cambridge, U.K.: Cambridge Univ. Press, 2005.

[30] R. A. Brockbank and C. A. A. Wass, "Non-linear distortion in transmission systems," J. Inst. Elect. Eng. III, Radio Commun., vol. 92, no. 17, pp. 45-56, Jul. 1945

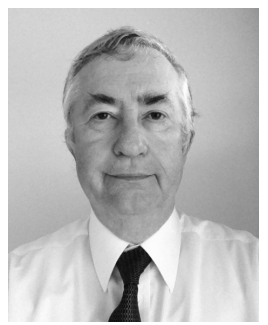

R. Allan Belcher (SM'86) received the B.Sc. degree in electronic engineering from the University of North Wales Bangor, Bangor, U.K., in 1969, and the $\mathrm{Ph} . \mathrm{D}$. degree in electronic engineering from the University of Surrey, Guildford, U.K., in 1977.

He was a Research Engineer with the BBC Engineering Research Department, London, U.K., from 1969 to 1978 , where he produced the patents for the DCF method. He became a Faculty Member with Swansea University, Swansea, U.K., where he originated patents related to digital audio conversion. Since 2005, he has been a Professor of Conversion Technology with Cardiff University, Cardiff, U.K., and has undertaken research into ADC/DAC systems for wide-band wide-dynamic range sensors.

Dr. Belcher originated and chaired the IEE series of the international conferences on advanced ADCs, DACs, and their applications, served in the IEEE, British Standards Institution, and IEC Standards Committees related to ADCs, and is the Chairman of the IEC Working Group responsible for IEC 60748-4-3. 